Bangladesh J. Plant Taxon. 25(1): 19-43, 2018 (June)

(C) 2018 Bangladesh Association of Plant Taxonomists

\title{
TAXONOMIC REVISION OF SAUDI ARABIAN TETRAENA MAXIM. AND ZYGOPHYLLUM L. (ZYGOPHYLLACEAE) WITH ONE NEW VARIETY AND FOUR NEW COMBINATIONS
}

\author{
Dhafer Ahmed Alzahrani ${ }^{1}$ and Enas Jameel Albokhari ${ }^{2}$ \\ Department of Biological Sciences, Faculty of Science, King Abdulaziz University, \\ Jeddah, Saudi Arabia
}

Keywords: Taxonomic revision; Tetraena; Zygophyllum; New variety; New combination; Saudi Arabia.

\begin{abstract}
The genera Tetraena Maxim. and Zygophyllum L. (Zygophyllaceae) present different morphological characters, viz. growth habit, leaf features, flower traits and fruit shape, and have a high diversity of species in Africa, Australia and Asia. Six species of Tetraena [T. alba (L.f.) Beier \& Thulin, T. coccinea (L) Beier \& Thulin, T. decumbens (Delile) Beier \& Thulin, T. hamiensis (Schwein f.) Beier \& Thulin, T. propinqua (Decne.) Ghaz. \& Osborne and T. simplex (L.) Beier \& Thulin], and one species of Zygophyllum (Z. fabago L.) have been identified in Saudi Arabia, most of which grow in sandy soils and saline habitats as shrubs and herbs. One new endemic variety (T. alba var. arabica Alzahrani \& Albokhari) along with four new combinations [T. alba var. amblyocarpa (Baker) Alzahrani \& Albokhari, T. hamiensis var. qatarensis (Hadidi ex Beier \& Thulin) Alzahrani \& Albokhari, T. hamiensis var. mandavillei (Hadidi ex Beier \& Thulin) Alzahrnai \& Albokhari, and T. propinqua subsp. migahidii (Hadidi ex Beier \& Thulin) Alzahrani \& Albokhari] are proposed. Descriptions, illustrations, distribution maps and a key for identification of the taxa are presented. Conservation status has been proposed for the new variety and combinations.
\end{abstract}

\section{Introduction}

The genus Zygophyllum L. distributed in Saudi Arabia received much attention based on morphological and anatomical characters (Hadidi, 1978; Migahid, 1978, 1996; Hosny, 1988; Mandaville, 1990; Chaudhary, 2001; Soliman et al., 2010; Waly et al., 2011). However, according to the most recent taxonomic proposal of Tetraena Maxim. and Zygophyllum presented by Beier et al. (2003), the most Saudi Arabian taxa of Zygophyllum were transferred to Tetraena. Zygophyllum and Tetraena have similar morphological characters, viz. growth habit, leaf features, flower traits and fruit shape. Beier et al. (2003) showed that Zygophyllum and Tetraena could easily be distinguished from each other by the characters of fruit dehiscence and staminal appendages. Zygophyllum propinquum was not included in Beier et al. (2003) and it was not mentioned as a synonym under any species in their study. However, Ghazanfar and Osborne (2015) transferred this species to Tetraena propinqua.

Taxonomically, Zygophyllaceae R. Br. was placed in different orders by several authors. Engler (1964) placed the family in the order Geraniales, while Cronquist (1968) and Hutchinson (1969) positioned Zygophyllaceae in the order Sapindales. Dahlgren (1980) deposited it in the order Geraniales, following Engler (1964), but Takhtajan (1980) positioned the family within Rutales. Currently, APG III (2009) placed Zygophyllaceae with Krameriaceae within a new order

${ }^{1}$ Corresponding author. Email: dalzahrani@kau.edu.sa; dhaferalzahrani@hotmail.com

${ }^{2}$ Department of Biological Sciences, Faculty of Applied Sciences, Umm Al-Qura University, Makkah, Saudi Arabia. 
Zygophyllales. These two families are placed with strong support as sister to a clade containing more than two orders. Sheahan and Chase (2000) analysed both $r b c \mathrm{~L}$ and $t r n \mathrm{~L}-\mathrm{F}$ sequences from 36 taxa of Zygophyllaceae and their results were supported by previous classification of Zygophyllaceae into the five subfamilies (Zygophylloideae, Larreoideae, Seetzenioideae, Tribuloideae and Morkillioideae). Moreover, Sheahan and Chase (2000) have indicated that Tetraena is nested within the large and variable Zygophyllum.

The genus Zygophyllum was first described by Linnaeus (1753) and has been accepted by several authors who worked broadly on systematics of this genus (Zumbruch, 1931; Van Huyssteen, 1937; Oltmann, 1971; Hadidi, 1978; Sheahan and Chase, 1996, 2000; Van Zyl, 2000). Linnaeus (l.c.) classified six species within Zygophyllum, namely Z. fabago L., Z. morgsana L., Z. sessilifolium L., Z. fulvum L., Z. coccineum L. and Z. spinosum L. Based on growth habit, androecium characters and dehiscence of the capsule Van Huyssteen (1937) split Zygophyllum into two subgenera, viz. Zygophyllotypus Huysst. (= subgenus Zygophyllum) and Agrophyllum, and further subdivided Zygophyllotypus into eight sections and Agrophyllum into five sections. In this classification, Zygophyllum coccineum, Z. album and Z. aegyptium were placed in section Mediterranea Engl., and Z. simplex and Z. decumbens in section Bipartita Huysst. Z. dumosum was placed in section Alata Huysst (Van Huyssteen, 1937).

Hadidi (1977) recognized eight species of Zygophyllum in Arabia and all of them belong to section Mediterranea, including two new species, i.e. Zygophyllum mandavillei Hadidi, and $Z$. migahidii Hadidi. Z. migahidii is closely related to Z. propinquum, but they differ in flower and fruit characters. In Z. migahidii, the flowers and fruit are solitary at each node, while they are grouped in clusters in Z. propinquum, conversely, Z. mandavillei can be easily recognized from other species of its section by its glabrous, large long-stalked flowers and sausage-shaped capsule. Later, Hadidi (1978) described Z. qatarense from Qatar as a new species. Hosny (1988) reported 13 species and three varieties of Zygophyllum in Arabia and among them10 species and one variety were distributed in Saudi Arabia. She classified them into two subgenera Zygophyllum and Agrophyllum (Necker) Endl. ex Van Huyssteen following Engler (1931) and Van Huyssteen (1937). According to Van Huyssteen (1937), two of the Saudi species belong to section Bipartita, seven species belong to section Mediterranea, including Z. boulosii A. Hosny as a new species, and two species belong to section Hamiensia Engl.

Sheahan and Chase (1996) studied the phylogenetic relationships of Zygophyllaceae based on morphology, anatomy and the $r b c \mathrm{~L}$ sequence and found Fagonia as sister to the rest of the subfamily, while Zygophyllum fabago (type species of Zygophyllum) is a sister to genus Augea, and $Z$. simplex is a sister to genus Tetraena and concluded that $Z$. simplex might not belong to Zygophyllum. Later, Sheahan and Chase (2000) investigated the phylogenetic relationships of 36 taxa of Zygophyllaceae including 15 species of Zygophyllum from Africa, Australia and southwest Asia using nucleotide sequences of the plastid gene $r b c \mathrm{~L}$ and non-coding trnL-F. Their results agreed with a high support to the previous results that stated Zygophyllaceae needs to be divided into five subfamilies and the subfamily Zygophylloideae was further classified into five clades with a high support of bootstrap. Also, they concluded that Zygophyllum is polyphyletic. Zygophyllum fabago was nested with another Asian species, Z. xanthoxylum, whereas Z. simplex was placed in a strong clade with genus Tetraena and other species of Zygophyllum: Z. cylindrifolium, Z. decumbens, Z. album and Z. coccineum (last three are distributed in Saudi Arabia). Moreover, molecular studies have indicated that Tetraena is nested within the large and paraphyletic Zygophyllum (Sheahan and Chase, 2000).

Van Zyl (2000) made a revision for 54 species of south African Zygophyllum and classified these species into two subgenera Zygophyllum and Agrophyllum, based on morphological characters, more particularly capsule dehiscence, seed attachment and presence of spiral threads in 
the seed mucilage. The result agreed with classification provided by Endlicher (1841) and Van Huyssteen (1937). Takhtajan (1987) separated the genus Tetraena from subfamily Zygophylloideae and erected Tetraenoideae based on morphology of pistil, fruit, pollen grains and chromosomes. Species within the genus Tetraena can be distinguished by growth habit, plant colour, leaf structure, flower colour, and fruit type and shape (Van Huyssteen, 1937; Hosny, 1988; Van Zyl, 2000; Chaudhary, 2001; Beier et al., 2003).

Based on trnL plastid DNA sequences and morphological characters Beier et al. (2003) showed that Zygophylloideae is monophyletic, whereas the genus Zygophyllum is paraphyletic, since it was placed with the genera of Augea Thunb., Tetraena and Fagonia L. In addition, they proposed a new classification for Tetraena and Zygophyllum which is supported by combination of morphological and molecular data, transferring 35 species from Zygophyllum to Tetraena as new combinations. These species are known from Africa and Asia. Zygophyllum is characterized by a loculicidal capsule and undivided staminal appendages, while Tetraena is distinguished by a schizocarp and sometimes bipartite staminal appendages. Subsequently, many authors agreed with this transfer and used the combinations proposed by Beier et al. (2003) as valid in their works (Norton et al., 2009; Louhaichi et al., 2011; Mosti et al., 2012; Sakkir et al., 2012; Azevedo, 2014; Symanczik et al., 2014).

Recently, Alzahrani and Albokhari (2017a) studied phylogenetic relationships of 44 specimens representing seven taxa of Saudi Arabian Tetraena Maxim. and Zygophyllum L., based on individual and combined chloroplast DNA data of $r b c \mathrm{~L}$ and $t r n \mathrm{~L}-\mathrm{F}$. Molecular phylogenetic of the cpDNA analysis of this study, divided Saudi Arabian Tetraena plants into six groups: $T$. hamiensis (Schweinf.) Beier \& Thulin, T. propinqua (Decne.) Ghazanfar \& Osborne, T. alba (L. f.) Beier \& Thulin, T. coccinea (L.) Beier \& Thulin, T. simplex (L. f.) Beier \& Thulin, and $T$. decumbens (Delile) Beier \& Thulin and one species of Zygophyllum (Zygophyllum fabago L.).

In Saudi Arabia, Zygophyllaceae is represented by eight genera including Balanites Del., Fagonia L., Nitraria L., Peganum L., Seetzenia R. Br., Tetraena Maxim., Tribulus L. and Zygophyllum L. (Collenette, 1985, 1998, 1999; Mandaville, 1990; Migahid, 1996; Chaudhary, 2001). The genus Tetraena is widespread in Saudi Arabia, while the genus Zygophylum is only represented by Z. fabago in northern parts of Saudi Arabia. In Saudi Arabia, a few taxonomic studies on the genera Tetraena and Zygophyllum using morphological and anatomical characters have been carried out (Soliman et al., 2010; Al-Arjany, 2011;Waly et al., 2011) and new combination have been made (Alzahrani, 2017; Alzahrani and Albokhari, 2017b,c). The objective of the present study is to revise the Saudi Arabian genera Tetraena and Zygophyllum belonging to the family Zygophyllaceae with detailed taxonomic notes.

\section{Materials and Methods}

The present revisionary study of Tetraena Maxim. and Zygophyllum L. in Saudi Arabia is based on extensive field survey, literature and analysis of more than 348 specimens, including types and images of types from different herbaria, viz. BM, CAI, CAIM, E, K, KAUH, KSU and RIY. Field surveys were carried out in c. 31 localities, between 2013 and 2014, and 72 collected samples were deposited in KAUH (King Abdulaziz University Herbarium, Jeddah, Saudi Arabia). The collected specimens were critically studied and examined, and identifications were confirmed using standard literature (Hosny, 1988; Chaudhary, 2001; Beier et al., 2003). In each case, several duplicate voucher specimens were made and these were complemented with fresh material preserved in $70 \%$ ethanol and stored for further research.

A total of 74 morphological traits were found effective to determinate species and new combinations from field collections and herbarium specimens. These characters, including both vegetative and reproductive features, were examined and scored using a Novex dissecting 
microscope and X10 hand lens. Differences between species, subspecies and varieties were supported by distribution maps, diagnostic traits placed in the identification key, and drawings, and evaluation of synonyms. The conservation status for the new variety and combinations was assessed following the guidelines of IUCN (IUCN, 2014).

\section{Results and Discussion}

Zygophyllum is represented by only one species in Saudi Arabia, Z. fabago. It clearly differs from Tetraena species by several morphological characters such as size, shape and colour of leaves, flowers and fruits. The present study reveals that $Z$. fabago is characterized by 2 -foliolate, flat leaves, up to $4 \mathrm{~cm}$ long, and $2.5 \mathrm{~cm}$ wide, creamy flowers, and loculicidal, oblong-cylindrical capsule, up to $3 \mathrm{~cm}$ long. This is congruent with Beier's results (Beier et al., 2003).

Based on the morphological traits and morphometric analysis (Alzahrani, 2017; Alzahrani and Albokhari, 2017b, c), this study reported six species of Tetraena (e.g. T. alba, T. coccinea, T. decumbens, T. hamiensis, T. propinqua and T. simplex) distributed in Saudi Arabia.

\section{Key to the Saudi Arabian species of Zygophyllum and Tetraena}

1. Fruit a loculicidal capsule; leaves 2-foliolate, flat, obovate-elliptic. Zygophyllum fabago

- Fruit a schizocarp; leaves simple, 1- or 2-foliolate, mostly cylindrical and fleshy, seldom flat.

2. Staminal appendages bipartite; stems and leaves glabrous. 3

- $\quad$ Staminal appendages undivided; stems and leaves mostly 4 pubescent.

3. Fruits obovoid, 5-lobed; flowers yellow; leaves simple, sessile, Tetraena simplex cylindrical, fleshy.

- $\quad$ Fruits obconical, 5-ridged; flowers white creamy; leaves 2- $\quad T$. decumbens foliolate, petiolate, obovate, flat.

4. Leaves 1-foliolate; fruits oblong-obovate, 5-angled at the upper end to cylindrical.

- Leaves 2-foliolate; fruits oblong-ovate, obconial or cylindrical, with or without ridges or lobes at the upper end.

5. Flowers arranged in clusters. Fruits obconical, 5-ridged at the upper end.

- $\quad$ Flowers 1-3 at each node.

6. Fruits cylindrical, without lobes or angled at the upper end (sausage shaped).

- $\quad$ Fruits ovate-oblong to obconical, 5-angled at the upper end.

T. hamiensis

Tetraena alba (L. f.) Beier \& Thulin, Pl. Syst. Evol. 240: 35 (2003).

(Fig. 1).

Diagnosis: $T$. alba differs from other species of the genus by its pubescent stem, 2-foliolate, cylindrical with acute apex, fleshy leaves, mostly arranged flowers in clusters, undivided staminal appendages and fruit shape. It occurs in western Saudi Arabia. Three morphological variations are recognized.

Small shrub, perennial, green or greenish grey, 50-60 cm tall, $40 \mathrm{~cm}$ wide. Stem pubescent, with unicellular simple trichomes. Leaves 2-foliolate, $7-12 \times 3.0-5.5 \mathrm{~mm}$, fleshy, cylindrical or elliptic, apex acute; petiole $10-18 \mathrm{~mm}$; stipules triangular, herbaceous, $1.0 \times 1.5 \mathrm{~mm}$, pubescent. Flowers arranged in clusters, sometimes solitary, bisexual, white, $4.0-5.5 \times 3-5 \mathrm{~mm}$, pedicel 1-2 $\mathrm{mm}$ long. Sepals 5, rounded-obtuse at the apex, herbaceous, yellowish green, obovate, $3-4 \times 2-3$ 
mm, pubescent, aestivation imbricate. Petals 5, white, spathulate, 3.5-6.0 $\times 1-2 \mathrm{~mm}$, aestivation valvate. Stamens 10, 3-4 mm long, staminal appendages undivided, 2.0-2.5×1.0 mm; anthers 2lobed, yellow, dorsifixed, dehiscent longitudinally; disc smooth. Ovary 5-locular, pubescent; style single, c. $1 \mathrm{~mm}$ long. Fruit a schizocarp, obconical, oblong- obconical or star shaped, acute, with keeled lobes 7-12×(2-6)8-13 mm, pubescent, pericarp extended as wings, peduncle 2-6 mm long, pubescent.

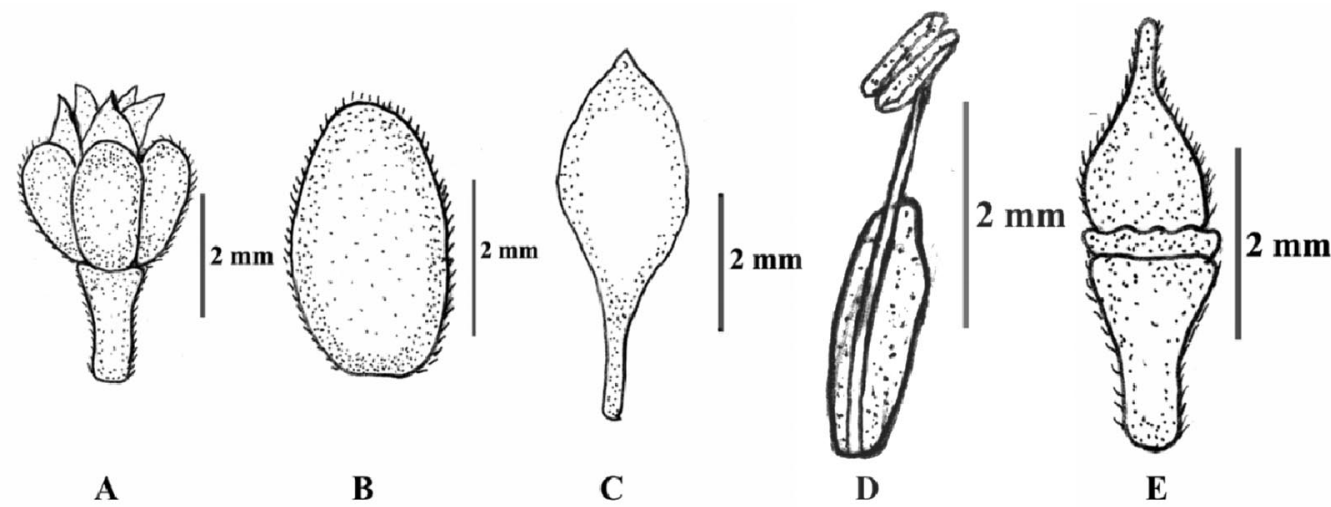

Fig. 1. Tetraena alba: A. Flower; B. Sepal; C. Petal; D. Stamen; E. Ovary (Alzahrani and Albokhari, 2017b).

Tetraena alba consists of three varieties, viz. T. alba (L. f.) Beier \& Thulin var. alba, T. alba (L.f.) Beier \& Thulin var. arabica Alzahrani \& Albokhari, var. nov. and T. alba (L. f.) Beier \& Thulin var. amblyocarpa (Baker) Alzahrani \& Albokhari, comb. nov. Taxonomy of these varieties are summarized below:

Tetraena alba (L. f.) Beier \& Thulin var. alba. Zygophyllum album L. f., Dec. Pl. Hort. Upsal.: 11 t. 6 (1762); Z. proliferum Forssk., Fl. Egypt. Arab.: 12 (1775).

(Figs 2A, D \& G).

Diagnosis: This variety can be distinguished by the petiole of the leaflets up to $15 \mathrm{~mm}$ long, flowers 4.0-4.5×3-4.5 mm; schizocarps obconical, star-shaped, with thick broad lobes 8-10 mm long, 7-10 $\mathrm{mm}$ wide of upper end, 3-6 $\mathrm{mm}$ wide of lower end, pedicel up to $3 \mathrm{~mm}$ long.

Type: Linnaeus HL544-2 [LINN, lectotype designated by El-Hadidi in Webbia 33: 51(1978)].

Vernacular names: Rotreyt, Qarmal, Harm.

Phenology: February to June.

Distribution: Saudi Arabia: Along the Red Sea coast (Fig. 3). Worldwide: Egypt, Jordan, Tunisia, Palestine, Somalia, South Africa and Greece.

Habitat: Found in the salt marshy habitats, coastal and inland saline sandy soils, dunes and sheets, and in saline depressions.

Specimens examined: SAUDI ARABIA: Shuaiba (2052'23"N 39²2'6"E), February 2013,

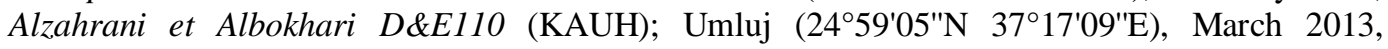
Alzahrani et Albokhari D\&E132, D\&E134, D\&E139 (KAUH); Umluj (2503'34.87"N 37²'50.86"E), May 2014, Alzahrani D148, D153 (KAUH); Coast $12 \mathrm{~km}$ north of Muweli (2741'6.02"N 35²9'20.33"E), September 1983, Collenette 4521 (RIY, K); near Umm Sidrah 75 $\mathrm{km}$ north of Jeddah, January 1980, Collenette 1518 (K). EGYPT: Sallum east, April 1932, Shabetai 1780 (CAIM); North of Helwan, February 1944, Davis 6302 B (E); Helwan, March 1891, 
Scott Elliot 3554 (E). JORDAN: Aqaba, October 1989, Leonard 7468 (E). TUNISIA: Monastir, August 1968, Davis 48050 (E); Southeast Tunisia, west of Oudref, February 1966, Archibald 884 (E). GREECE: July 1950, Davis 18109 (E); EP. Ierapetro, October 1966, Greuter 7811 (E).

Tetraena alba (L. f.) Beier \& Thulin var. arabica Alzahrani \& Albokhari, var. nov.

(Figs 2B, E \& H).

Diagnosis: This variety can be distinguished by its petiole of the leaflets, up to $18 \mathrm{~mm}$ long, flowers $5.5 \times 5.0 \mathrm{~mm}$, schizocarps oblong-obconical, star shaped, with narrow lobes, $11-13 \mathrm{~mm}$ long, upper end 8-10 $\mathrm{mm}$ wide, lower end 2-3 $\mathrm{mm}$ wide, pedicel up to $6 \mathrm{~mm}$ long.

Type: Saudi Arabia, Umluj (24 $\left.58^{\prime} 19^{\prime \prime N} 37^{\circ} 17^{\prime} 03^{\prime \prime} \mathrm{E}\right)$, March 2013, Alzahrani et Albokhari 138 (Holotype: KAUH; Isotype: KSU).

Small shrub, perennial, green or greenish grey, $50-60 \mathrm{~cm}$ tall, $40 \mathrm{~cm}$ wide. Stem pubescent, with unicellular simple trichomes. Leaves 2-foliolate, $7-12 \times 3.0-5.5 \mathrm{~mm}$, fleshy, cylindrical or elliptic, apex acute; petiole up to $18 \mathrm{~mm}$ long; stipules triangular, herbaceous, $1.0 \times 1.5 \mathrm{~mm}$, pubescent. Flowers white, arranged in clusters, sometimes solitary, bisexual, $5.5 \times 5.0 \mathrm{~mm}$, pedicel 1-2 mm long. Sepals 5, rounded-obtuse at the apex, herbaceous, yellowish green, obovate, 3-4×2$3 \mathrm{~mm}$, pubescent, aestivation imbricate. Petals white, 5, spathulate, $3.5-6.0 \times 1-2 \mathrm{~mm}$, aestivation valvate. Stamens $10,3-4 \mathrm{~mm}$ long, staminal appendages undivided, $2.0-2.5 \times 1 \mathrm{~mm}$; anthers 2 lobed, yellow, dorsifixed, dehiscent longitudinally; disc smooth. Ovary 5-locular, pubescent; style single, $1 \mathrm{~mm}$ long. Schizocarp oblong-obconical, star shaped, with narrow lobes, 11-13×(2-3)8$10 \mathrm{~mm}$, pubescent, pericarp extended as wings, peduncle pubescent, up to $6 \mathrm{~mm}$ long.

Vernacular names: Rotreyt, Qarmal, Harm.

Phenology: February to June.

Distribution: Endemic to Saudi Arabia and apparently restricted to its western cost, mainly in Umluj (Fig. 3).

Habitat: Found in coastal and inland saline sandy soils, and salt marshy areas.

Etymology: The varietal epithet is derived from Arabia, the area of its distribution.

Conservation status: Based on its known distribution (area of occupancy estimated to be less than $10 \mathrm{~km}^{2}$ ) and abundance (number of mature individuals less than 50), the IUCN Red List Category (IUCN, 2014) "Critically Endangered" is here attributed to this variety.

Tetraena alba (L. f.) Beier \& Thulin var. amblyocarpa (Baker) Alzahrani \& Albokhari, comb. nov. Zygophyllum amblyocarpum Baker, Hooker's Icon. Pl. 24: t. 2358 (1895); Z. amblyocarpum Baker, Kew Bull. 1894: 339 (1894), nom. nud.; Z. album L. f. var. amblyocarpum (Baker) ElHadidi in Webbia 33: 52 (1978); Z. album L. f. var. amblyocarpum (Baker) El-Hadidi in Bot. Not. 131: 441 (1978).

(Figs 2C, F\& I).

Diagnosis: This variety is recognized by petiole of leaflets up to $10 \mathrm{~mm}$ long, flowers $4 \times 4 \mathrm{~mm}$, schizocarps obconical, acute with keeled lobes 9-13 mm long, 8-12 mm wide at upper end, 2-3 $\mathrm{mm}$ wide at lower end and pedicel up to $6 \mathrm{~mm}$ long.

Type: Hadramout, Al Mukalla, Shary Burrock Valley, December 1893; Lunt 51 (Holotype: $\mathrm{K}$ !; Isotype: $\mathrm{BM})$.

Phenology: February to June.

Vernacular names: Rotreyt, Qarmal, Harm.

Distribution: Saudi Arabia: Shuaibah (Fig. 3). Worldwide: South Arabia (Yemen), tropical East and North Africa (Egypt). 
Habitat: Salt marshy areas.

Conservation status: Least Concern (lc), locally common on the west coast of Saudi Arabia, cost of Yemen, Egypt and Somalia.

T. alba var. alba
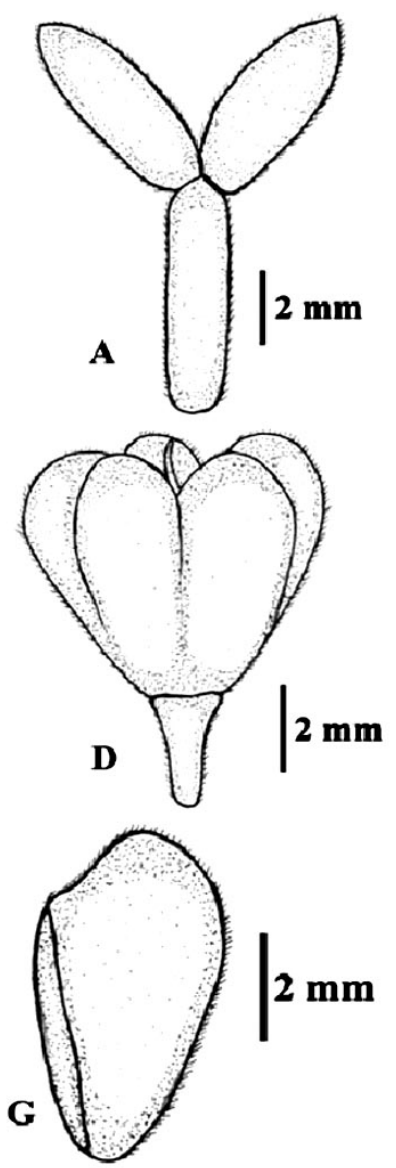

T. alba var. arabica
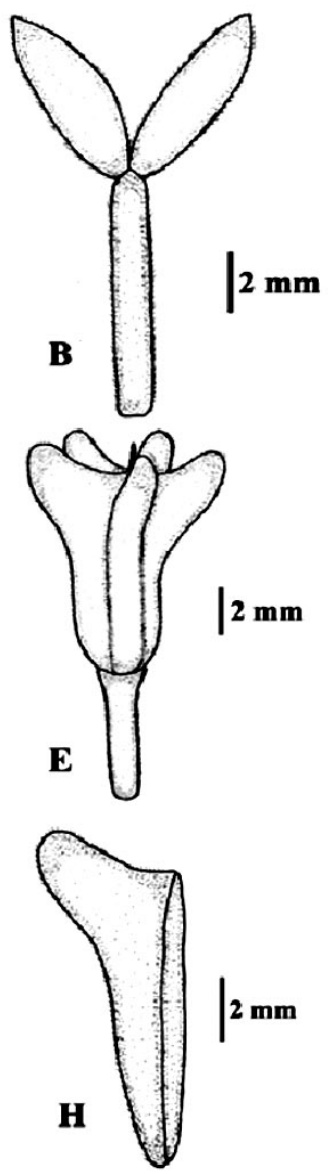

T. alba var. amblyocarpa
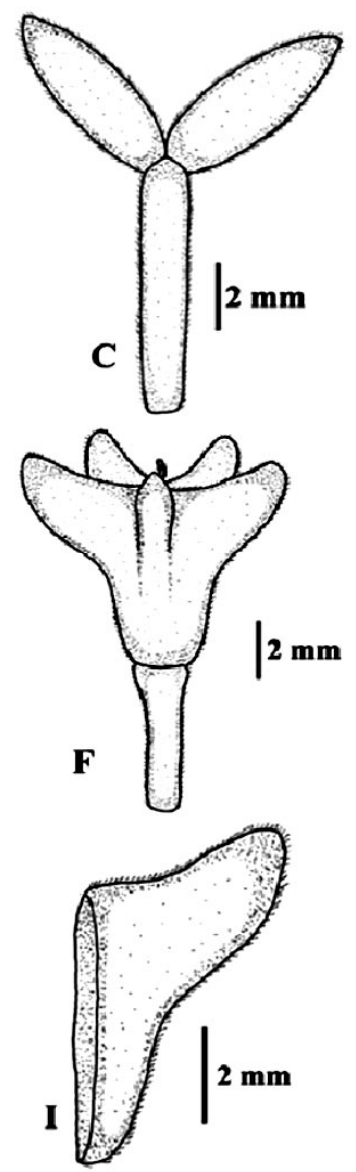

Fig. 2. Variations in morphological characters of Tetraena alba varieties: A. Leaf of T. alba var. alba;

B. Leaf of T. alba var. arabica; C. Leaf of T. alba var. amblyocarpa; D. Fruit of T. alba var. alba; E. Fruit of T. alba var. arabica; F. Fruit of T. alba var. amblyocarpa; G. Schizocarp lobe of T. alba var. alba; H. Schizocarp lobe of T. alba var. arabica; I. Schizocarp lobe of T. alba var. amblyocarpa (Alzahrani and Albokhari, 2017b).

Specimens examined: SAUDI ARABIA: Shuaiba (2051'10"N 39²3'47"E), February 2013, Alzahrani et Albokhari D\&E107 (KAUH). YEMEN: Hadramout, Al Mukalla, Shary Burrock valley, December 1893, Lunt 51 (K!, holotype); Hadramout, $81 \mathrm{~km}$ from Qusayir along road to Sayhut, October 1992, Thulin et al. 8247 (K). EGYPT: Jamailia, February 1948, Shabetai 7730 (CAIM); Red Sea region, May 2005, Abdel-Ghani et Abdel-Fattah s.n. (CAIM); Safaga, May 2005, Abdel-Ghani et Abdel-Fattah s.n. (CAIM). 


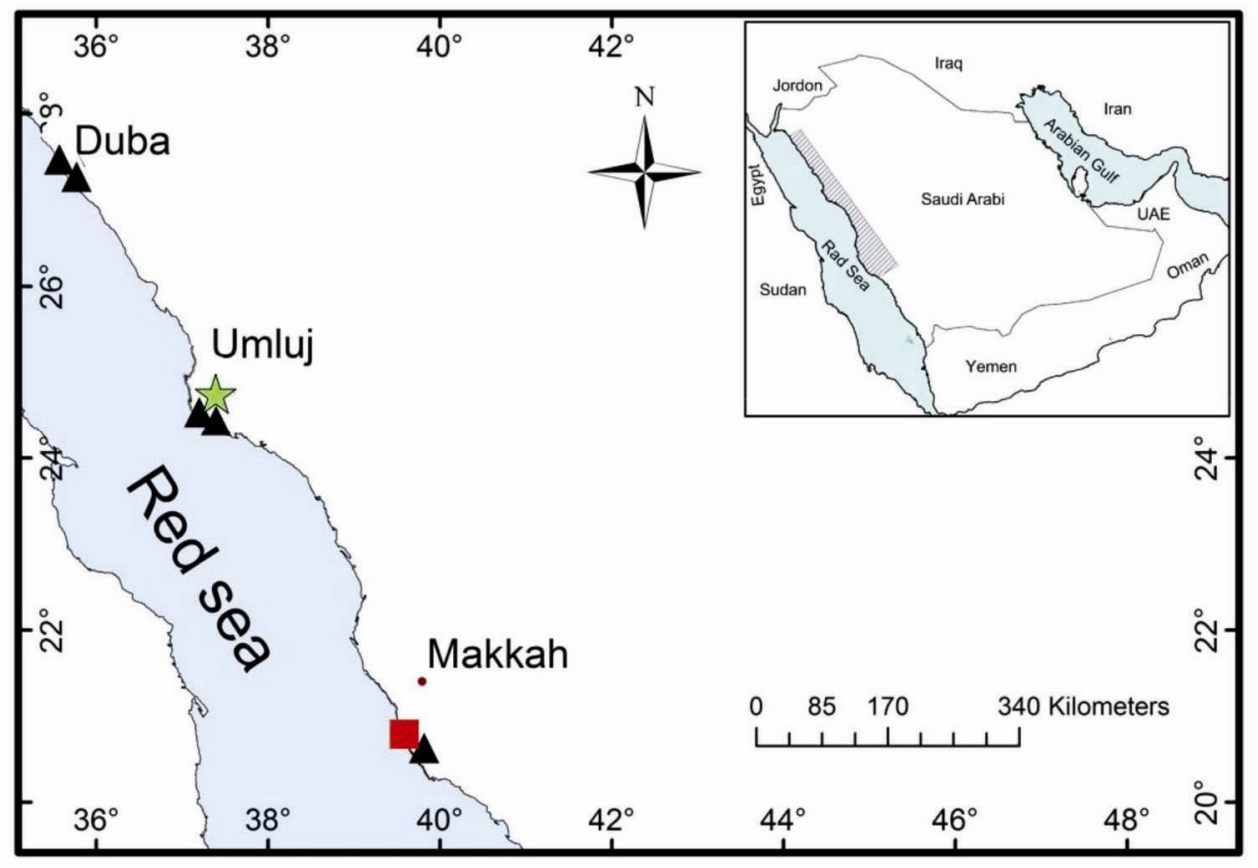

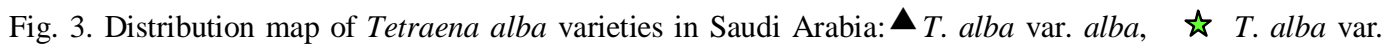
arabica, T. alba var. amblyocarpa (Alzahrani and Albokhari, 2017b).

Tetraena coccinea (L) Beier \& Thulin, Pl. Syst. Evol. 240: 35 (2003). Zygophyllum coccineum L., Sp. Pl. 1: 386 (1753); Z. desertorum Forssk., Fl. Aegypt.-Arab.: 87 (1775); Z. berenicense Scweinf., Fl. Egypt III: 65 (1887); Z. coccineum L. var. berenicense (Schweinf.) Muschl., Man. Fl. Egypt 1: 578 (1912).

(Fig. 4).

Diagnosis: $T$. coccinea with a shrubby habit can be recognized by its cylindrical fruits and persistent triangular stipules.

Type: Inter Kahiram \& Sués, August 1762, Forsskål s.n. (Holotype: C; Isotype: BM! LD).

Small shrubs, perennial, green, up to $75 \mathrm{~cm}$ tall and $100 \mathrm{~cm}$ wide. Stem pubescent, with unicellular simple trichomes. Leaves 2-foliolate, cylindrical, up to $14.0 \times 4.5 \mathrm{~mm}$, fleshy, petiole up to $20 \mathrm{~mm}$ long; stipules triangular, herbaceous, $1.5 \times 1.0 \mathrm{~mm}$, pubescent. Flowers bisexual, white, 4-7×4-5 mm, pedicel up to $10 \mathrm{~mm}$ long. Sepals 5 , rounded-obtuse at the apex, herbaceous, yellowish green, obovate, 4-6×2-3 mm, pubescent, aestivation imbricate. Petals 5, white, spathulate, 5-7×2.0-2.5 mm, aestivation valvate. Stamens 10, 3.0-4.5 mm long, staminal appendages undivided, 2-3×1.0-1.5 mm; anthers 2-lobed, yellow, dorsifixed, dehiscent longitudinally; disc smooth. Ovary 5-locular, pubescent; style single, $1 \mathrm{~mm}$ long. Fruit a schizocarp, cylindrical, $9-12 \times 3-6 \mathrm{~mm}$, glabrous, peduncle up to $11 \mathrm{~mm}$ long.

Vernacular names: Harm, Rotreyt, Batbat.

Phenology: February to June.

Distribution: Saudi Arabia: North-west to south-west Saudi Arabia (Fig. 5). Worldwide: Kuwait, Yemen, East and North Africa, and Palestine. 


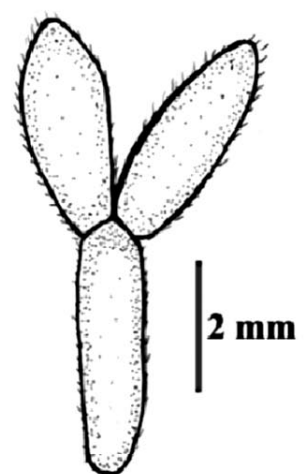

A

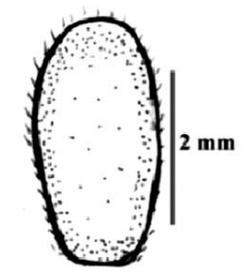

D

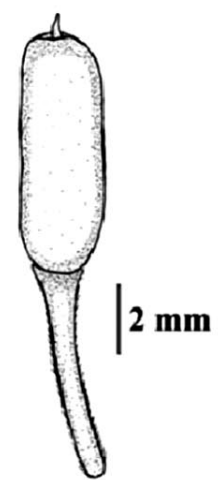

B

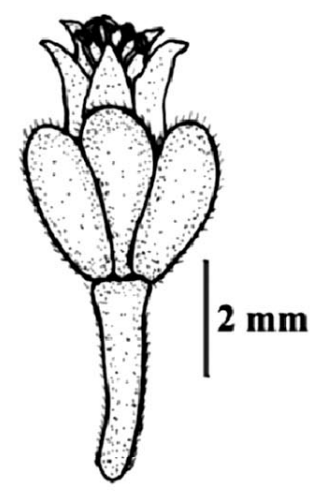

C

Fig. 4. Tetraena coccinea: A. Leaf; B. Fruit; C. Flower; D. Sepal; E. Petal; F. Stamens.

Habitat: Found in salt marshy areas.

Specimens examined: SAUDI ARABIA: South of Jeddah (22 $\left.31^{\prime} 11^{\prime \prime N} 39^{\circ} 10^{\prime} 41^{\prime \prime E}\right)$, February 2013, Alzahrani et Albokhari D\&E101, D\&E102, D\&E104, D\&E105 (KAUH); Shuaibah $\left(20^{\circ} 52^{\prime} 23^{\prime \prime N} 39^{\circ} 22^{\prime} 16 " \mathrm{E}\right)$, February 2013, Alzahrani et Albokhari D\&E108, D\&E11, D\&E113 (KAUH); North of Jeddah $\left(21^{\circ} 50^{\prime} 23^{\prime \prime N} 39^{\circ} 07^{\prime} 05^{\prime \prime} E\right)$, February 2013, Alzahrani et Albokhari $D \& E 114, D \& E 115, D \& E 116, D \& E 117, D \& E 118$ (KAUH); South of Alleith (19'56'15"N 40³1'17"E), February 2013, Alzahrani D\&E119, D\&E120 (KAUH); near Rabigh (2331'35"N 38 40'27"E), March 2013, Alzahrani et Albokhari D\&E123, D\&E124, D\&E125, D\&E126 (KAUH); between Rabigh and Yanbu $\left(23^{\circ} 53^{\prime} 08^{\prime \prime N} 38^{\circ} 27^{\prime} 08^{\prime \prime E}\right)$, March 2013, Alzahrani et

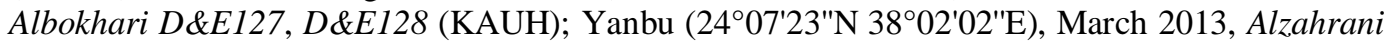
et Albokhari D\&E129, D\&E130, D\&E131, D\&E135, D\&E136, D\&E140, (KAUH); Farasan Island, February 1986, Collenette 10357 (RIY); Tabouk, September 1983, Chaudhary H8251 (RIY); Duqm Sabkha, February 1999, Someya et Wutaid H19036 (RIY); Oasis near Duba, February 1999, Someya et Wutaid H19035 (RIY); Rabigh, May 1998, Someya et WutaidH19037 (RIY); Dumsaq, June 1988, Chaudhary H14159 (RIY); North Hijaz, May 1978, Collenette 747 (K); Red Sea near Jeddah, October 1983, Collenette 5491 (K, E); Yanbu al Bahr, 1972, Collenette 72-203 (K); Jabal Ohod north of Madinah, February 1945, Khattab 323 (CAI). EGYPT: The desert road between Cairo-Faiyum at $40 \mathrm{~km}$, August 1972, Abbas et Abdel-Hay 838 (CAIM); Wadi Hamad, April 1944, Davis 7165 (E); Lower Wadi Digla, October 1944, Davis 7805 (E). YEMEN: January 1979, Wood 2676 (K, E); Meidi, March 1944, Khattab 681 (CAIM). 


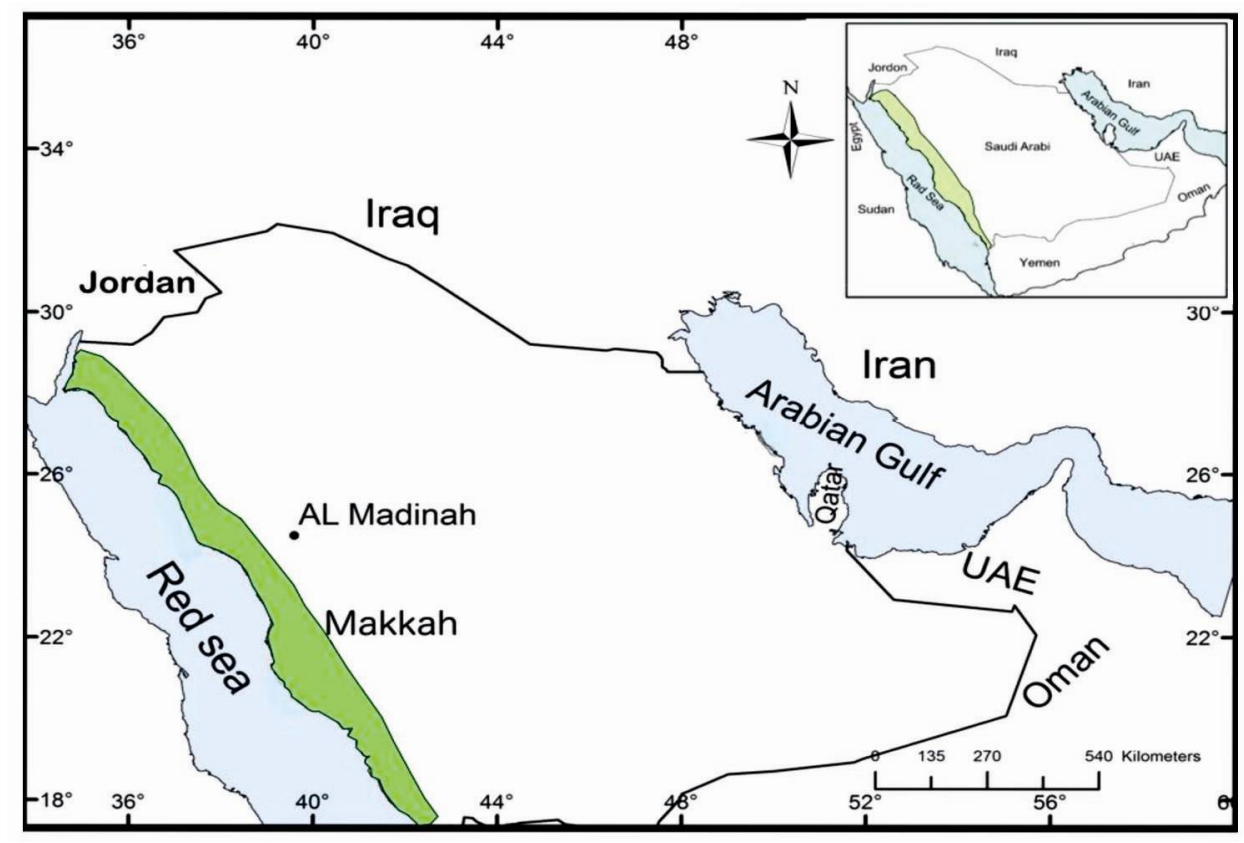

Fig. 5. Distribution map of Tetraena coccinea in Saudi Arabia.

Tetraena decumbens (Delile) Beier \& Thulin, Pl. Syst. Evol. 240: 35 (2003). Zygophyllum decumbens Delile, Descr. Egypte, Hist. Nat. :221, t. 27, fig. 3 (1813); Z.decumbens Delile var. megacarpum Hosny, Bot. Not. 130: 467-468 (1977).

(Fig. 6).

Diagnosis: T. decumbens can be distinguished from other Tetraena species by its shrubby habit, 2-foliolate flat leaves, white creamy flowers, bipartite staminal appendages and glabrous stem. It is sympatric to T. alba and T. coccinea in western Saudi Arabia, but these species have pubescent stem, undivided staminal appendages and different fruit features.

Type: Valée dans I'Egaroment; Delile 6967 (Holotype: MPU; Isotype: Fl).

Small shrub, perennial, green, $50 \mathrm{~cm}$ tall, $100 \mathrm{~cm}$ wide. Stem glabrous. Leaves 2-foliolate, obovate, flat, up to $21 \times 12 \mathrm{~mm}$, apex rounded, fleshy, petiole $12-15 \mathrm{~mm}$ long; stipules triangular, $1.0 \times 1.5 \mathrm{~mm}$, glabrous. Flowers bisexual, white-creamy, 5-6×4-5 mm, pedicel up to $7 \mathrm{~mm}$ long. Sepals 5, rounded-obtuse at the apex, herbaceous, yellowish green, obovate, glabrous, $3 \times 2 \mathrm{~mm}$, aestivation imbricate. Petals 5, white-creamy, spathulate, $4.0 \times 1.5 \mathrm{~mm}$, aestivation valvate. Stamens 10, 4.0-4.5 mm long, staminal appendages bipartite, $1.5 \times 0.5 \mathrm{~mm}$; anthers 2-lobed, yellow, dorsifixed, dehiscent longitudinally; disc smooth. Ovary 5-locular, glabrous; style single, c. $2 \mathrm{~mm}$ long. Fruit a schizocarp, obconical, 5-ridged, 3-6×1.5-5.0 mm, glabrous, peduncle up to $15 \mathrm{~mm}$ long.

Vernacular names: Harm, Rotreyt, Qarmal, Batbat.

Phenology: February to June.

Distribution: Saudi Arabia: Western to north-western region (Fig. 7). Worldwide: Oman, Yemen, Egypt, Sinai, Sudan, Somalia, Eritrea, and South Africa.

Habitat: Growing in sandy and gravels habitat. 


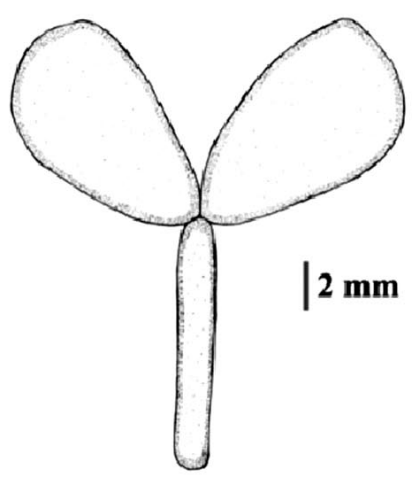

A

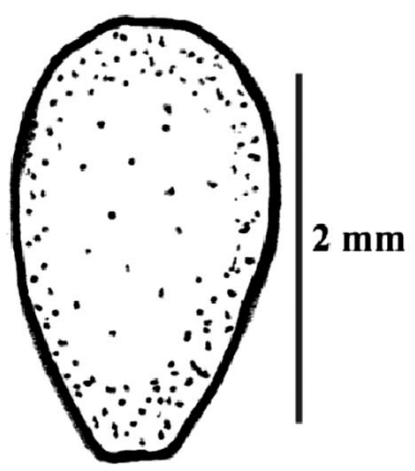

D

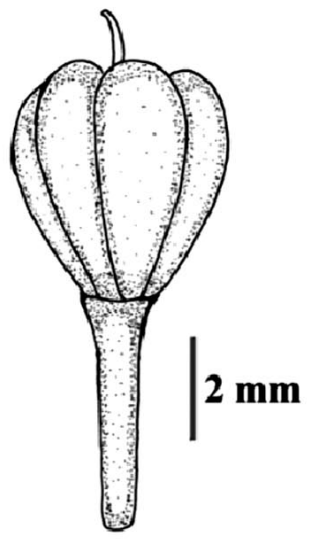

B

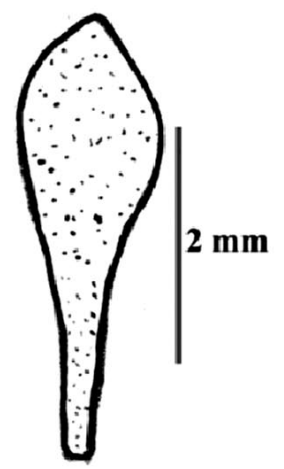

$\mathbf{E}$

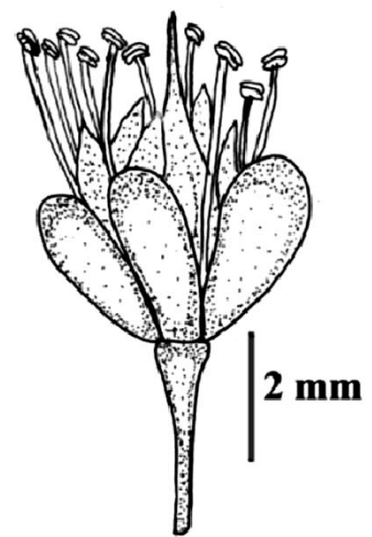

C

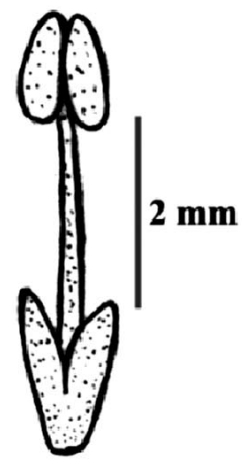

F

Fig. 6. Tetraena decumbens: A. Leaf; B. Fruit; C. Flower; D. Sepal; E. Petal; F. Stamen.

Specimens examined: SAUDI ARABIA: $30 \mathrm{~km}$ south of Umluj $\left(24^{\circ} 45^{\prime} 06^{\prime \prime} \mathrm{N} 37^{\circ} 19^{\prime} 56^{\prime \prime} \mathrm{E}\right)$, March 2013, Alzahrani et Albokhari D\&E142 (KAUH); Al Wajh (26²0'36.6"N 36²3'13.5"E), May 2014, Alzahrani D147, D152 (KAUH); Wadi Al Bayda, February 1998, Someya et Wutaid H19029 (RIY); Jabal Hassan W coast, July 1998, Someya et Wutaid H19028 (RIY); North of Muweli between Duba and Ash Sharma, September 1983, Collenette 8846 (RIY); Jaziat Qummaah, February 1998, Someya et Wutaid H19041 (RIY); Duba north of Hedjaz, January 1944, Khattab K33 (CAI); 12 km north of Muweli Ash Sharma road, September 1983, Collenette 4519 (K). OMAN: Dhofar, $25 \mathrm{~km}$ south of Thumrait on Salalah road, September 1984, Miller 7654 (K); Ayun road $5 \mathrm{~km}$ east of turnoff to pools, September 1985, Miller 7654 (K); Jabal Qamar $5 \mathrm{~km}$ northwest of Janook, October 1979, Miller 2621 (K). YEMEN: Shabwah, $2 \mathrm{~km}$ northeast of Mahfis Wadi Bottom, October 1992, Thulin et al. 7981 (K); Shabwa Wadi $5 \mathrm{~km}$ south of Ataq, January 1988, Rowaished et al. 2792 (K); Hadramout, Central Plateau $19 \mathrm{~km}$ south of Sayun along the road to Al Mukalla, June 1987, Boulos et al.17055 (K). EGYPT: Jabal Araqa, March 1944, Davis 9799 (RIY); Wadi Quseib north Galala, March 1964, Boulos s.n. (K); in the desert of Elsaff south of Helwan, April 1959, Boulos s.n. (K). 


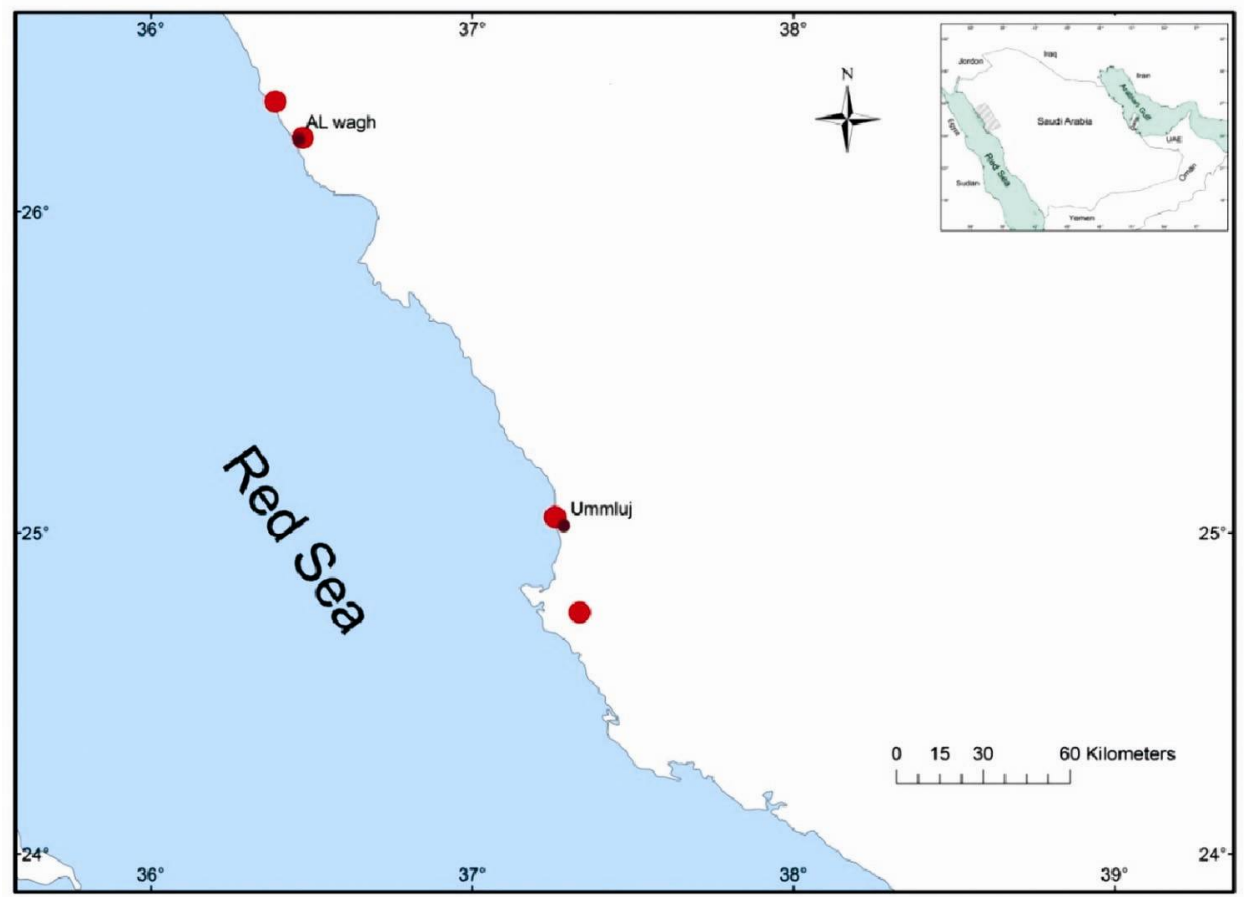

Fig. 7. Distribution map of Tetraena decumbens in Saudi Arabia.

Tetraena hamiensis (Schweinf.) Beier \& Thulin, Pl. Syst. Evol. 240: 35 (2003).

(Fig. 8).

Diagnosis: T. hamiensis can mostly be recognized by the presence of 1-foliolate leaves, but when they are 2-foliolate, usually are fleshy, terete or globular, pubescent stem, solitary white flowers and undivided staminal appendages.

Small shrubs, perennial, green, reddish or yellowish green, up to $80 \mathrm{~cm}$ tall, $90 \mathrm{~cm}$ wide. Stem pubescent, with unicellular simple trichomes. Leaves mostly 1-foliolate, sometimes 2-foliolate in upper branches, terete, globular, cylindrical or clavate, $4-9 \times 3-6 \mathrm{~mm}$, fleshy, pubescent or glabrous, petiole equal or longer than leaflets, up to $9 \mathrm{~mm}$ long; stipules triangular, herbaceous, $1.0 \times 1.5 \mathrm{~mm}$, pubescent. Flowers bisexual, solitary at each node, white, $4-6 \times 3-5 \mathrm{~mm}$, pedicel 3-5 $\mathrm{mm}$ long. Sepals 5, rounded-obtuse at the apex, herbaceous, yellowish green, obovate, 3-5 $\times 2-3$ $\mathrm{mm}$, pubescent, aestivation imbricate. Petals 5, white, spathulate, 4-6×1.5-2.0 mm, aestivation valvate. Stamens 10, 2-5 mm long, staminal appendages undivided, $2-3 \mathrm{~mm}$ long, $1.0-1.5 \mathrm{~mm}$ wide; anthers 2-lobed, yellow, dorsifixed, dehiscent longitudinally; disc smooth. Ovary 5-locular, pubescent; style single, $0.5-1.5 \mathrm{~mm}$ long. Fruit a schizocarp, oblong-obovate, oblong-obconical, 5 -angled or cylindrical, $8-20 \times(2-3.5) 2-5 \mathrm{~mm}$, pubescent or glabrous, peduncle 5-10 $\mathrm{mm}$ long, pubescent or glabrous.

T. hamiensis comprises three varieties, namely, T. hamiensis (Scweinf.) Beier \& Thulin var. hamiensis, T. hamiensis (Schweinf.) Beier \& Thulin var. qatarensis (Hadidi ex Beier \& Thulin) Alzahrani \& Albokhari, comb. nov. and T. hamiensis (Schweinf.) Beier \& Thulin var. mandavillei (Hadidi ex Beier \& Thulin) Alzahrnai \& Albokhari, comb. nov. 

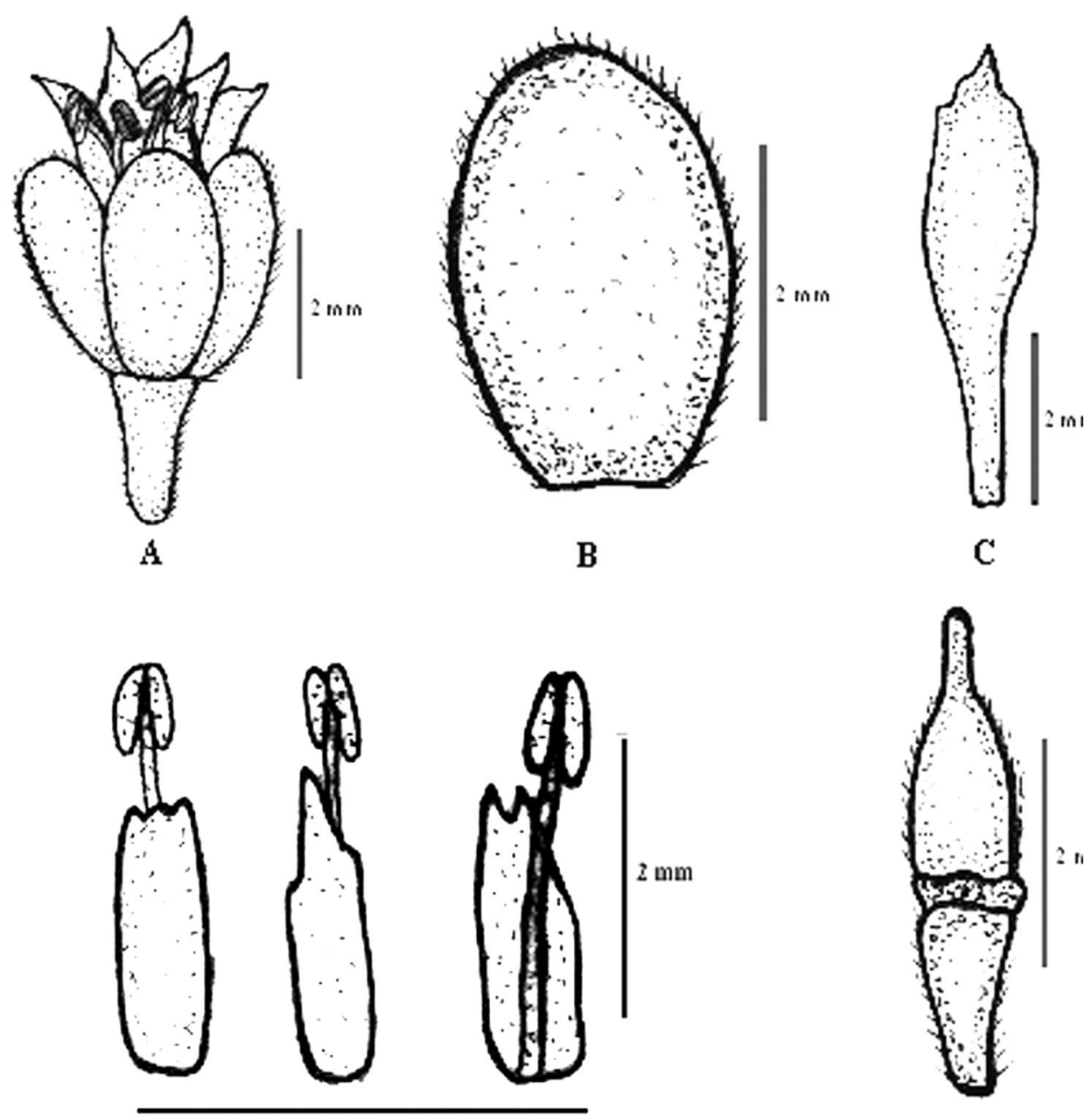

Fig. 8. Tetraena hamiensis: A. Flower; B. Sepal; C. Petal; D. Stamens; E. Ovary (Alzahrani and Albokhari, 2017c).

Tetraena hamiensis (Scweinf.) Beier \& Thulin var. hamiensis. Zygophyllum hamiense Schweinf., Bull. Herb. Boissier VII. App. II : 277 (1899); T. hamiensis (Schweinf.) Beier \& Thulin, Pl. Syst. Evol. 240: 36 (2003).

(Figs 9A \& D).

Diagnosis: T. hamiensis var. hamiensis is distinguished by its green, clavate, $6-9 \times 3-5 \mathrm{~mm}$ leaflets, petiole up to $9 \mathrm{~mm}$, pedicel up to $5 \mathrm{~mm}$ long, schizocarp oblong-obconical, 5-angled, clearly lobed, $10-13 \times 3-4 \mathrm{~mm}$, pubescent, peduncle up to $10 \mathrm{~mm}$ long.

Type: El Hami, east Schehr. Schweinfurth 182 (Isotype: W).

Vernacular name: Harm.

Phenology: February to June and September to November.

Distribution: Saudi Arabia: Eastern and south-central region of Saudi Arabia (Fig. 10). Worldwide: United Arab Emirates, Oman, Kuwait, Yemen, Iran and Somalia.

Habitat: In sandy and saline soils. 
Conservation status: T. hamiensis var. hamiensis appears to be distributed in some localities in the Eastern and south-central region of Saudi Arabia. At the international level, this variety is evaluated as Least Concern (LC) since it also grows in United Arab Emirates, Oman, Kuwait, Yemen, Iran and Somalia (IUCN, 2014).

Specimens examined: SAUDI ARABIA: Al Ahsa, Qatar road (25'16'30"N 49 41'09"E), May 2013, Alzahrani D18 (KAUH); Al Ahsa, Qatar road (2449'54"N 5040'25"E), $25 \mathrm{~km}$ before Salwa, May 2013, Alzahrani D19 (KAUH); Al Ahsa, Qatar road, $10 \mathrm{~km}$ before Alaudaidah (24²7'32"N 51 02'52"E), May 2013, Alzahrani D24 (KAUH); Al Ahsa, Dammam road (253'33"N 49³1'11"E), May 2013, Alzahrani D28 (KAUH); Alqateef, Alsharqia, July 1997, Atar 5723 (KSU); Aflag, Layla, August 1998, Atar 5834 (KSU); Dhahran, December 1953, Baker XI (K). UNITED ARAB EMIRATES: West side of jabal Hafit, January 1983, Brown 439 (CAI). OMAN: Nizwa Agricult Inst. Firg., November 1981, Maconochie 2948 (K); Bahala, March 1976, Radcliffe-Smith 3790 (K); Dhufar, $50 \mathrm{~km}$ west of Mudhai, September 1985, Miller 7621 (K). YEMEN: Hadramout, Sayun outside the town, weeds in field and road sides, June 1987, Boulos et al. 17042 (CAI); Wadi Hajr, $100 \mathrm{~km}$ west of Mukalla, Howtah $11 \mathrm{~km}$ north of Meifa Haga, February 1989, Miller et al. 8153 (K, E). IRAN: Southeast Iran, Zahedan province, 24 miles of Rask road to Chah Bahar, March 1971, Grey-Wilson et Hewer 262 (K).

Tetraena hamiensis (Schweinf.) Beier \& Thulin var. qatarensis (Hadidi ex Beier \& Thulin) Alzahrani \& Albokhari, comb. nov. Zygophyllum qatarense Hadidi, Webbia 32 (2): 394 (1978); Z. hamiense var. qatarense (Hadidi) Thomas \& Chaudhary, Flora of the Kingdom of Saudi Arabia 2: 502 (2001); T. qatarensis (Hadidi) Beier \& Thulin, Pl. Syst. Evol. 240: 36 (2003).

(Figs 9B \& E).

Diagnosis: T. hamiensis var. qatarensis is distinguished by its reddish or olive green, globular, 4-6×4-6 mm leaflets, petiole up to $8 \mathrm{~mm}$, pedicel up to $3 \mathrm{~mm}$ long, schizocarp oblong-obovate, 5-angled, $8-10 \times 2-3 \mathrm{~mm}$, pubescent, partly lobed, peduncle up to $7 \mathrm{~mm}$ long.

Type: Qatar, Um Slal Ali, c. 25 km north of Doha, March 1977, Boulos 10953 (Holotype: K!; Isotype: $\mathrm{CAI} \& \mathrm{Fl})$.

Vernacular name: Harm.

Phenology: February to June and September to November.

Distribution: Saudi Arabia: Eastern region and north-central part of Saudi Arabia (Fig. 10). Worldwide: Qatar, Kuwait, Bahrain, United Arab Emirates, Oman, Socotra, Samha Isl., Abd-alKuri Isl. (Yemen) and Iraq.

Habitat: Found in the saline sand, including beaches, coastal areas, and rocky habitat.

Conservation status: T. hamiensis var. qatarensis appears to be distributed in some localities in the eastern and north-central region of Saudi Arabia. At the international level, this variety is evaluated as Least Concern (LC) since it also grows in Qatar, Kuwait, Bahrain, United Arab Emirates, Oman, Socotra, Samha Isl., Abd-al-Kuri Isl. (Yemen) and Iraq (IUCN, 2014).

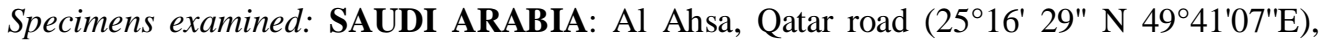
May 2013, Alzahrani D16 (KAUH); Al Ahsa, Qatar road (2448'40"N 5044'26"E), May 2013, Alzahrani D20, D21, D22 (KAUH); Buraidah, March 1997, Alfarhan et Thomas 766 (KSU); AlAhsa, March 1996, Thomas 766 (KSU); Aljubail, Alsharqia, July 1997, Atar $H 5729$ (KSU); Alsafaneiyah, Dammam, February 1981, Migahid et Alsheikh s.n. (KSU, H19992); Umm Assahik, Alsharqia, July 1997, Atar H5748 (KSU); Rocky coastal area near Batha check point, Salwa region, March 1990, Chaudhary et al. H13357 (RIY); Dareen Island, May 1987, Chaudhary $H 12190$ (RIY); Abqaiq-Hofuf road 87 km from Dhahran, April 1982, Podzorski 811 (RIY); 
Nairyah, October 1983, Jeha H8711 (RIY); 18 km north of Dammam, February 1982, Naylor 5 (E). QATAR: Um slal Ali, c. $25 \mathrm{~km}$ north of Doha, March 1977, Boulos 10953 (K!, holotype); Dukham Camp, 12 m waste ground, December 1970, Wilcox 38 (K); Sheikh Khalifa Ibn Ali Al Thani Garden, April 1977, Boulos 11179 (K). KUWAIT: Al-Khiran, March 1983, Rawi et al. 1550 (CAI); Roadsides between Al-Ahmadi and mina Abdullah, March 1995, Mathew 2531 (K). BAHRAIN: Near base of central hills of Bahrain main island, April 1984, Rezk 103 (K); Jerdab, 1985, Naguib 404 (K); Al-Areen Wild Life Park and Reserve, April 1985, Boulos et Hasan 15687 (K). UNITED ARAB EMIRATES: Abu Dhabi, March 1981, Western BW 20 (K). OMAN: Wahiba sands, January 1986, Cope 36 (K); Nr Zukayt 10 km south-west of Izki, September 1979, Miller et Whitcombe 2017 (K); Kuria Muria Island, Al Hallaniyah Island, February 1993, McLeish 1587 (E). IRAQ: $25 \mathrm{~km}$ south-east of Zubair, March 1957, Ghiust et al. 16871 (K); 25 km southeast of Zubair, March 1957, Ghiust, Rawi et Rechinger 16872 (K); between Zubair and Safwan, March 1966, Alizzi 34353 (K).

Tetraena hamiensis (Schweinf.) Beier \& Thulin var. mandavillei (Hadidi ex Beier \&Thulin) Alzahrnai \& Albokhari, comb. nov. Zygophyllum mandavillei Hadidi, Publ. Cairo Univ. Herb. 78: 327 (1977); Z. hamiense var. mandavillei (Hadidi) Thomas \& Chaudhary, Flora of the Kingdom of Saudi Arabia 2: 502 (2001); T. mandavillei (Hadidi) Beier \& Thulin, Pl. Syst. Evol. 240: 36 (2003).

(Figs 9C \& F).

Diagnosis: T. hamiensis var. mandavillei is distinguished by its yellowish green, cylindrical, $7-$ $9 \times 3-5 \mathrm{~mm}$ glabrous leaflets, petiole equal to the leaflet, up to $9 \mathrm{~mm}$ long, pedicel up to $4 \mathrm{~mm}$ long, schizocarp cylindrical, oblong, 16-20×3-4 mm, glabrous, peduncle up to $5 \mathrm{~mm}$ long.

Type: Saudi Arabia, ArRub' al-Khali, Camp Shaybah 9, June 1970, Mandaville, 2892 (Holotype: BM!; Isotype: CAI).

Vernacular name: Harm.

Phenology: February to June and September to November.

Distribution: Saudi Arabia: Central, eastern, north and eastern ArRub' al-Khali, Doshak Island, and south-west region of Saudi Arabia (Fig. 10). Worldwide: Oman, United Arab Emirates and Yemen (Aden Desert).

Habitat: Red sands, gravels or saline areas.

Conservation status: On the current evidence T. hamiensis var. mandavillei appears to be distributed in Eastern and Southwest region, north-west, northern and eastern of ArRub al Khali of Saudi Arabia. This species is evaluated as Least Concern (LC) since it also grows in Oman, United Arab Emirates and Yemen (IUCN, 2014).

Specimens examined: SAUDI ARABIA: Khurais, Al Ahsa road (25 $\left.13^{\prime} 55^{\prime \prime} \mathrm{N} 48^{\circ} 36^{\prime} 16^{\prime \prime} \mathrm{E}\right)$,

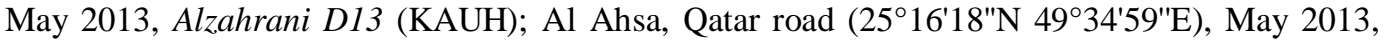

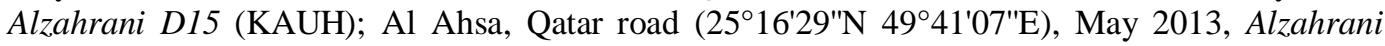
D17 (KAUH); Al Ahsa, Qatar road $25 \mathrm{~km}$ before Alaudaidah $\left(24^{\circ} 32^{\prime} 55^{\prime \prime N} 50^{\circ} 54^{\prime} 16^{\prime \prime} \mathrm{E}\right)$, May 2013, Alzahrani D23 (KAUH); Al Ahsa, Qatar road, $10 \mathrm{~km}$ before Alaudaidah $\left(24^{\circ} 27^{\prime} 32^{\prime \prime} \mathrm{N}\right.$ $51^{\circ} 02^{\prime} 52^{\prime \prime}$ ), May 2013, Alzahrani D25 (KAUH); Al Ahsa, Qatar road, Alaudaidah (24⒉ $26^{\prime} 07^{\prime \prime} \mathrm{N}$ $\left.51^{\circ} 07^{\prime} 01 " \mathrm{E}\right)$, May 2013, Alzahrani D26 (KAUH); Shedgum, next to the cement factory, Al AhsaDammam road (2540'07"N 49³0'31"E), May 2013, Alzahrani D30 (KAUH); Wadi Baysh, near Sabiya, June 1999, Alfarhan et al. H19742 (KSU); Near Shabita $\left(22^{\circ} 13^{\prime} \mathrm{N} 54^{\circ} 17^{\prime} \mathrm{E}\right)$, February 1990, Chaudhary et al. H13312 (RIY); Doshak Island, June 1988, Chaudhary H15762 (RIY); Layla lakes, sol Layla, March 1987, Collenette 6046 (RIY, K); 10 km north-west of campus S-3, north-eastern ArRub' al-Khali, February 1979, Mandaville 7085 (E); ArRub' Al-Khali, Camp Shaybah 9, June 1970, Mandaville 2892 (BM!, holotype). OMAN: Near Wadi Tawsinat, north 
Dhofar, May 1982, Gallagher 6464/26 (E). UNITED ARAB EMIRATES: Sweehan, February 1996, Boer 103 (RIY).

\section{T. hamiensis var. hamiensis}

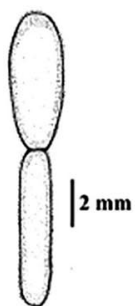

A

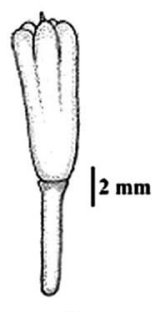

D
T. hamiensis var. qatarensis

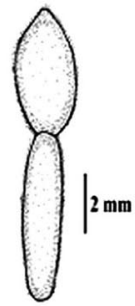

B

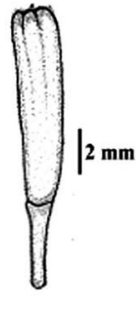

E
T. hamiensis var. mandavillei

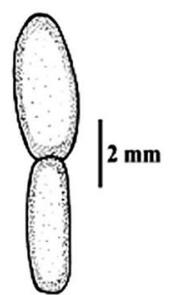

c

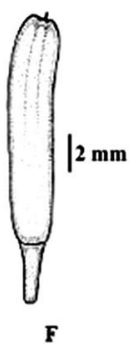

Fig. 9. Variation in morphological characters of Tetraena hamiensis varieties: A. Leaf of T. hamiensis var. hamiensis; B. Leaf of T. hamiensis var. qatarensis; C. Leaf of T. hamiensis var. mandavillei; D. Fruit of T. hamiensis var. hamiensis; E. Fruit of T. hamiensis var. qatarensis; F. Fruit of T. hamiensis var. mandavillei (Alzahrani and Albokhari, 2017c).

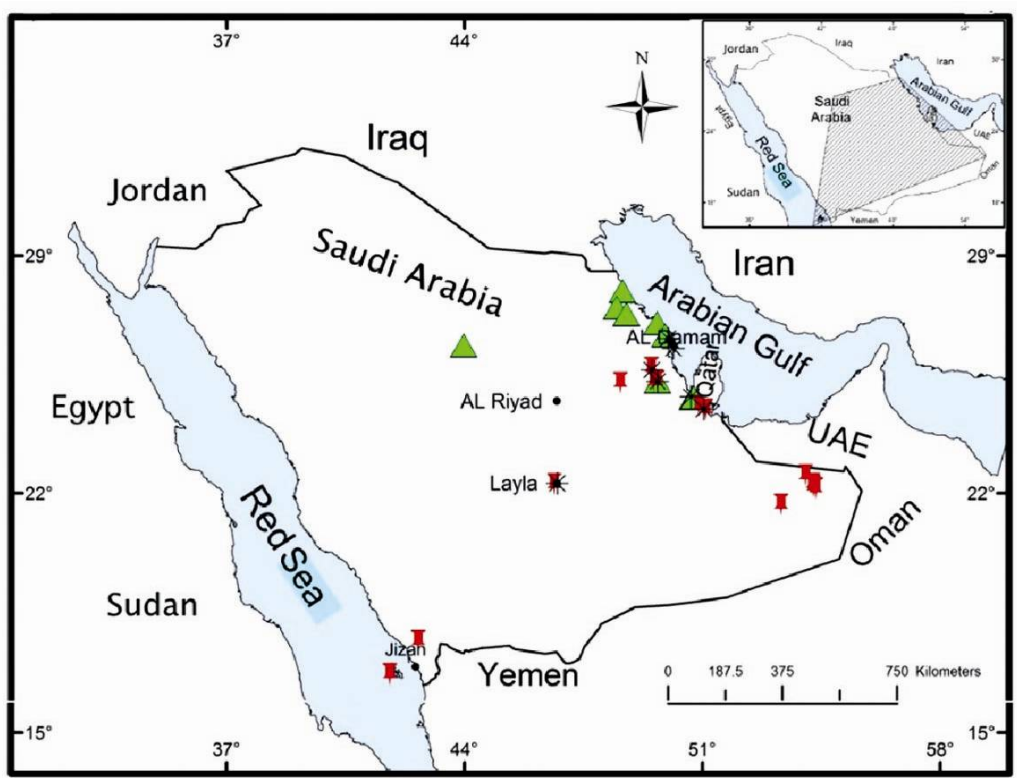

Fig. 10. Distribution map of Tetraena hamiensis varieties in Saudi Arabia. $\triangle$ T. hamiensis var. hamiensis, * T. hamiensis var. qatarensis, \$ T. hamiensis var. mandavillei (Alzahrani and Albokhari, 2017c). 
Tetraena propinqua (Decne.) Ghaz. \& Osborne, Kew Bull. 70: 38 (2015).

(Fig. 11).

Small shrubs, perennial, green, $50 \mathrm{~cm}$ tall, $80-100 \mathrm{~cm}$ wide. Stem pubescent, with unicellular simple trichomes. Leaves 2 -foliolate, cylindrical, up to $12 \times 4 \mathrm{~mm}$, apex rounded or acute, fleshy, pubescent, petiole up to $14 \mathrm{~mm}$ long; stipules triangular, herbaceous, $1.0 \times 1.5 \mathrm{~mm}$, pubescent. Flowers bisexual, white or white-creamy, $1-3$ at each node, $4-7 \times 3.5-5.0 \mathrm{~mm}$, pedicel 7-14 mm long. Sepals 5, rounded-obtuse at the apex, herbaceous, yellowish green, obovate, $3-5 \times 2-3 \mathrm{~mm}$, pubescent, aestivation imbricate. Petals 5, white, spathulate, $2.5-6.0 \times 1-3 \mathrm{~mm}$, aestivation valvate. Stamens 10, 3-5 mm long, staminal appendages undivided, 2.0-3.5 mm long, $1 \mathrm{~mm}$ wide; anthers 2-lobed, yellow, dorsifixed, dehiscent longitudinally; disc smooth. Ovary 5-locular, pubescent; style single, 1-2 mm long. Fruit a schizocarp, ovate to oblong or obconical, 5-angled, 7-13 $\times 2.0$ $6.5 \mathrm{~mm}$, pubescent, peduncle up to $14 \mathrm{~mm}$ long, pubescent.

\section{Key to the subspecies of Tetraena propinqua}

1. Leaflets apex acute; pedicel up to $7 \mathrm{~mm}$ long; schizocarp ovate oblong; peduncle up to $7 \mathrm{~mm}$ long.

- Leaflet apex rounded; pedicel up to $14 \mathrm{~mm}$ long; schizocarp subsp. migahidii obconical; peduncle up to $14 \mathrm{~mm}$ long.

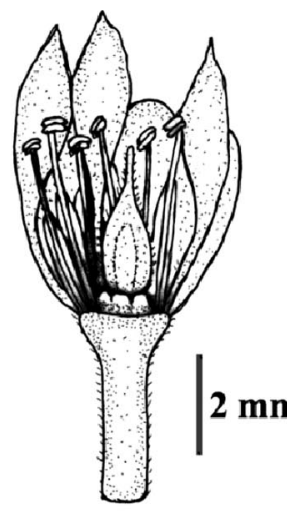

A

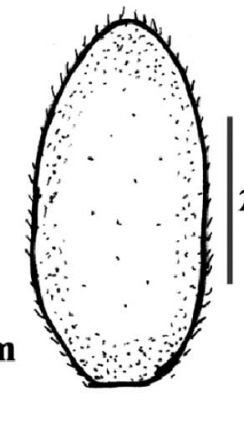

B

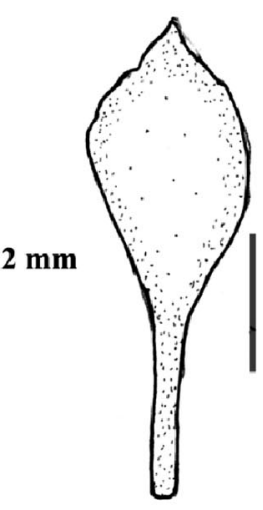

C

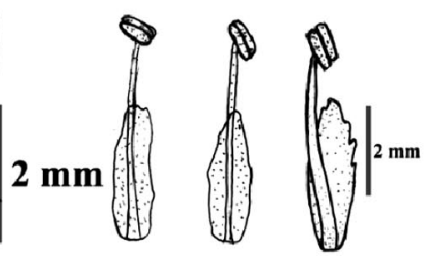

D

Fig. 11. Tetraena propinqua: A. Flower; B.Sepal; C. Petal; E. Stamens (Alzahrani, 2017).

Tetraena propinqua (Decne.) Ghaz. \& Osborne subsp. propinqua. Zygophyllum propinquum Decne., Ann. Sci. Nat., Bot. Sér. 2, 3: 283 (1835).

(Figs 12A, C \& E).

Diagnosis: T. propinqua subsp. propinqua can be distinguished by its acute leaflet apex, white flowers, up to $7 \mathrm{~mm}$ long pedicel, fruits ovate to oblong, 5- angled, 9-13 $\mathrm{mm}$ long, 4.0-6.5 mm wide at upper end, 2-4 $\mathrm{mm}$ wide at lower end, peduncle up to $7 \mathrm{~mm}$ long.

Type: Sinai, Gallam, Tor, June 1832, Bovè 172 \& 173 (Isotype: K!).

Vernacular names: Harm, Rotreyt.

Phenology: February to June.

Distribution: Saudi Arabia: Western to north-western Saudi Arabia (Fig. 13). Worldwide: Egypt, Sinai, Palestine, Iraq, Iran, Afghanistan, Pakistan and India.

Habitat: In sandy and gravel desert. 
Specimens examined: SAUDI ARABIA: Shuaibah $\left(20^{\circ} 52^{\prime} 23^{\prime \prime N} 39^{\circ} 22^{\prime} 16^{\prime \prime E}\right)$, February 2013, Alzahrani et Albokhari D\&E109 (KAUH); Umluj (24⒌ $\left.59^{\prime} 05^{\prime \prime N} 37^{\circ} 17^{\prime} 09^{\prime \prime E}\right)$, March 2013, Alzahrani et Albokhari D\&E133, D\&E137, D\&E141 (KAUH); Dhallam, May 1998, Thomas 5866 (KSU); $9 \mathrm{~km}$ south of Khaybar, October 1989, Collenette 7287 (K). IRAQ: On the road near Karbala-Liwa, July 1962, Al-Ani et Mohamed 12 (K); Karbala Musseiyib, May 1947, Gillett 9968 (K). EGYPT: Sinai, Tor, April 1836, Bove 274,275 (Isotype: K!); Gallam, Tor, June 1832, Bové 172, 173 (Isotype: K!).

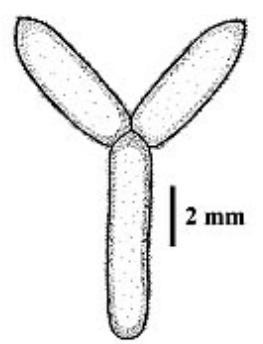

A

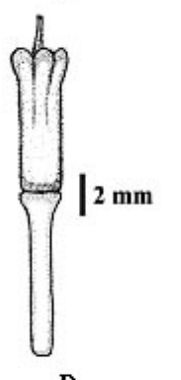

D

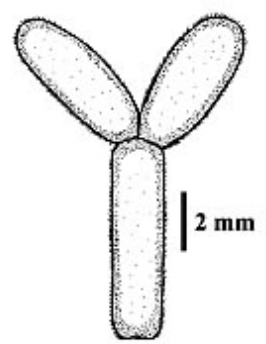

B

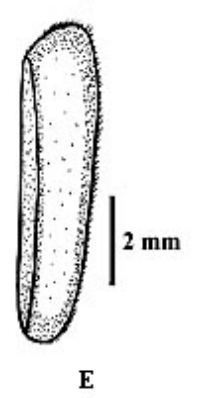

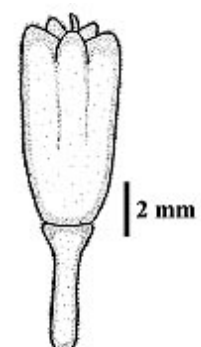

C

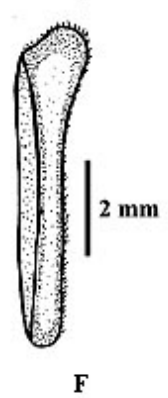

Fig. 12. Variation in morphological characters of Tetraena propinqua subspecies: A. Leaf of T. propinqua subsp. propinqua; B. Leaf of $T$. propinqua subsp. migahidii; C. Fruit of $T$. propinqua subsp. propinqua; D. Fruit of $T$. propinqua subsp. migahidii; E. Schizocarp lobe of $T$. propinqua subsp. propinqua; F. Schizocarp lobe of T. propinqua subsp. migahidii (Alzahrani, 2017).

Tetraena propinqua (Decne.) Ghaz. \& Osborne subsp. migahidii (Hadidi ex Beier \& Thulin) Alzahrani \& Albokhari, comb. nov. Zygophyllum migahidii Hadidi, Publ. Cairo Univ. Herb. 7 \& 8: 328 (1977); Z. propinquum subsp. migahidii (Hadidi) Thomas \& Chaudhary, Flora of the Kingdom of Saudi Arabia 2: 501 (2001); T. migahidii (Hadidi) Beier \& Thulin, Pl. Syst. Evol. 240: 36 (2003).

(Figs 12B, D \& F).

Diagnosis: T. propinqua subsp. migahidii is recognized by its rounded apex of the leaflet, whitecreamy flowers, pedicel up to $14 \mathrm{~mm}$ long, schizocarps obconical, 5-angled, 9-12 mm long, 3-5 $\mathrm{mm}$ wide at upper end, $2-5 \mathrm{~mm}$ wide at lower end, peduncle up to $14 \mathrm{~mm}$ long.

Type: Saudi Arabia, Al-Hail, Migahid, El-Sheikh et S. Awad 574/A (Holotype: CAI; Isotype: $\mathrm{KSU}$ ).

Vernacular names: Harm, Rotreyt.

Phenology: February to June.

Distribution: Saudi Arabia: North Saudi Arabia: Nafud Desert, West-central Saudi Arabia: Nejd Desert and Eastern Saudi Arabia (Fig. 13). Worldwide: Iraq.

Habitat: Sandy salt habitats and gravels desert. 
Specimens examined: SAUDI ARABIA: Alkhasrah, Taif-Riyadh road (23 $24^{\prime} 59^{\prime \prime} \mathrm{N}$ 4343'27"E), May 2013, Alzahrani D5 (KAUH); Almuzahmeiah, West of Riyadh (24²5'38"N 4557'32"E), May 2013, Alzahrani D6 (KAUH); Khurais Road, $150 \mathrm{~km}$ before Al Ahsa

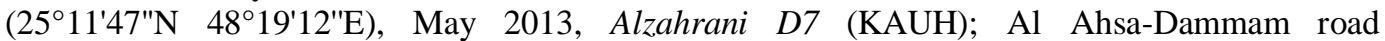
(253'3"N 49³2'12"E), May 2013, Alzahrani D27 (KAUH); Shedgum, next to the cement

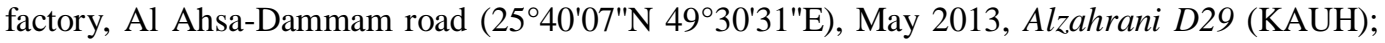
Buqaiq, Al Ahsa-Dammam road (26⒌'03"N 49 50'09"E), May 2013, Alzahrani D31 (KAUH); Riyadh King Khaled International Airport road (2450'57"N 46 44'14"E), May 2013, Alzahrani

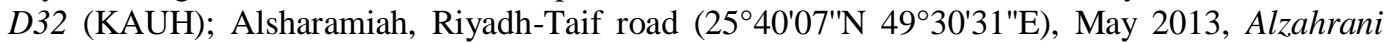

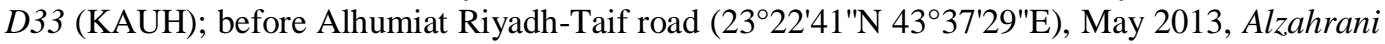

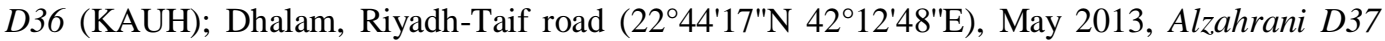
(KAUH); Beirut Street, Hail (27³3'48"N 41 $\left.{ }^{\circ} 43^{\prime} 47^{\prime \prime E}\right)$, May 2013, Alzahrani D53 (KAUH); Wadi

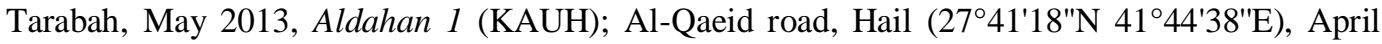
2013, Asiril (KAUH); Al-Hail, May 1976; Migahid et al. 574/A (Holotype: CAI; Isotype: KSU!); Riyadh, March 1993, Thomas 1253 (KSU); Al-Kharj road, April 1981, Noor 2296 (KSU); Buraidah, May 1983, Chaudhary H7832 (RIY); Unaizah, May 1978, Chaudhary s.n. (RIY); Chara, May 1985, Heemstra 7428 (RIY); Sulayyil, May 1996, Chaudhary H14228 (RIY); Riyadh, May 1984, Chaudhary H8356 (RIY); Aflaj, June 1984, Jahangir H8398 (RIY); RAWRC, 1984, Chaudhary H8489 (RIY); Aarqah, May 1984, Chaudhary 8355 (RIY); 30 km southwest Harad, November 1987, Mandaville 8696 (CAI); Southern of Riyadh, October 1987, Collenette 6314 (K, E); 2 km south east of Khurmah, Riyadh road, July 1991, Collenette 7851 (K). IRAQ. Habbanya, June 1966, Rawi et Alizzi 34453 (K); $40 \mathrm{~km}$ south of Baghdad, road to Karbala, November 1958, Rawi 26883 (K).

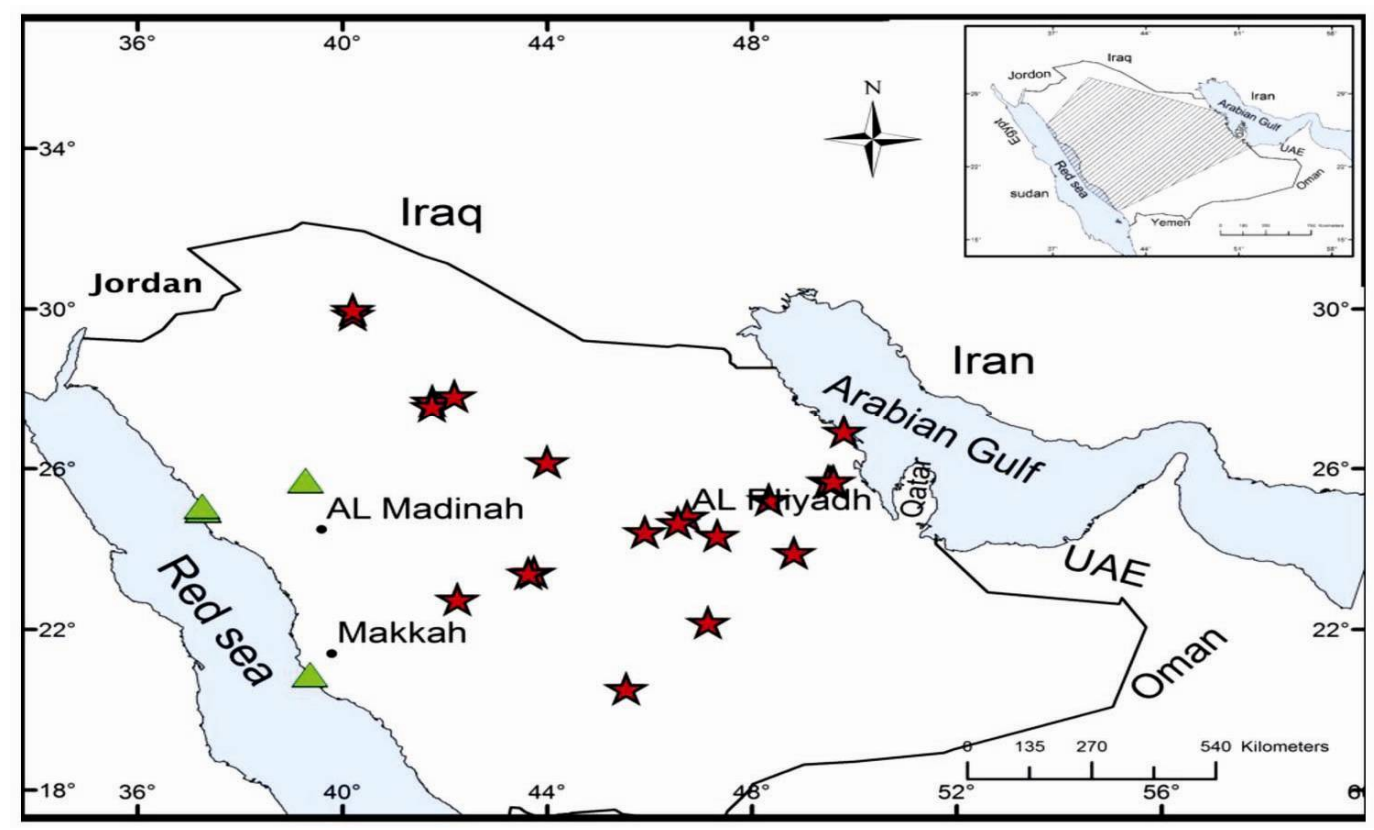

Fig. 13. Distribution map of Tetraena propinqua subspecies in Saudi Arabia. $\triangle T$. propinqua subsp. propinqua, T. propinqua subsp. migahidii (Alzahrani, 2017). 
Tetraena simplex (L.) Beier \& Thulin, Pl. Syst. Evol. 240: 36 (2003). Zygophyllum simplex L., Mant. Pl.: 68 (1767); Z. portulacoides Forssk., Fl. Egypt. Arab.: 88 (1775).

(Fig. 14).

Diagnosis: T. simplex is an annual herb and differs from other Tetraena species by its simple, opposite and sessile leaves, yellow flowers, bipartite staminal appendages and 5-lobed obovoid fruits.

Type: Egypt 1762-1763; Forsskål s.n. (Holotype: C; Isotype: LD Herb. Retzius).

Herbs, annual, green, 10-30 cm tall, 50-70 cm wide. Stem glabrous. Leaves simple, opposite, up to $20 \times 2.5 \mathrm{~mm}$, sessile, cylindrical, fleshy; stipules triangular, membranous, $1 \times 1 \mathrm{~mm}$, glabrous. Flowers bisexual, yellow, 2-4×3-5 mm, pedicel 1-2 mm long. Sepals 5, rounded-obtuse at the apex, herbaceous, yellowish green, obovate, $2 \times 1 \mathrm{~mm}$, aestivation imbricate. Petals 5 , yellow, spathulate, longer than sepals, $2.5-3.0 \times 1.0-1.5 \mathrm{~mm}$, aestivation valvate. Stamens 10, $2.5-3.0 \mathrm{~mm}$ long, appendages bipartite, hyaline, $1.0 \times 0.1-0.3 \mathrm{~mm}$, anthers 2-lobed, yellow, dorsifixed, dehiscent longitudinally; disc smooth. Ovary 5-locular, glabrous; style single, 1-3 mm long. Fruit a schizocarp, obovoid, 5-lobed, glabrous, 2-3 $\times 1.5-3.0 \mathrm{~mm}$, peduncle 1-2 $\mathrm{mm}$ long.

Vernacular names: Harm, Om thoreyb, Hamd, Qarmal.

Phenology: February to June and September to November.

Distribution: Saudi Arabia: Widely distributed throughout the country. Worldwide: Arabian Peninsula, United Arab Emirates, Oman, Yemen, Iran, Jordan, Palestine, Pakistan, India, and tropical Africa (Ghazanfar, 2007).

Habitat: Grows in sandy soils.

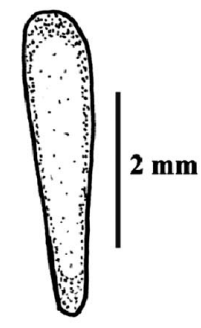

A

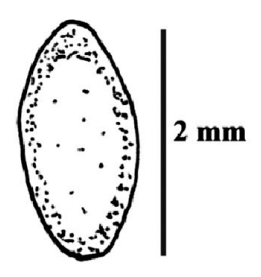

D

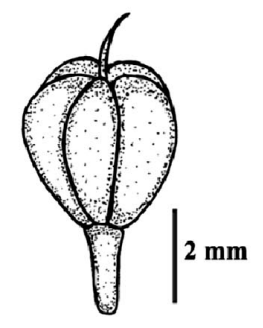

B

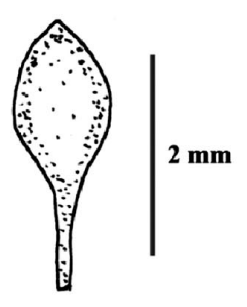

$\mathbf{E}$
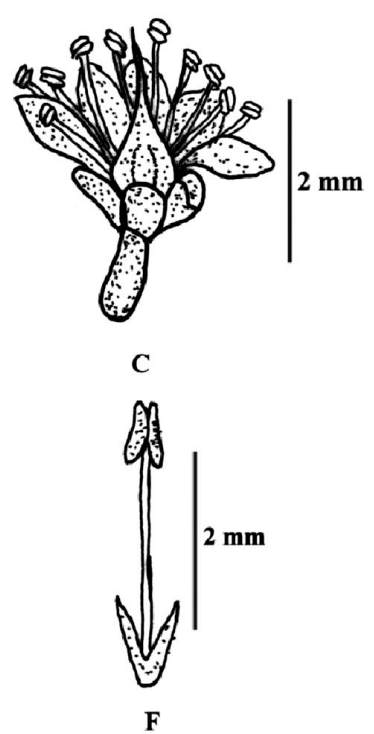

Fig. 14. Tetraena simplex: A. Leaf; B. Fruit, C. Flower; D. Sepal; E. Petal; F. Stamen.

Specimens examined: SAUDI ARABIA: Dhalam, Taif Riyadh road $\left(22^{\circ} 12^{\prime} 10^{\prime \prime} \mathrm{N}\right.$

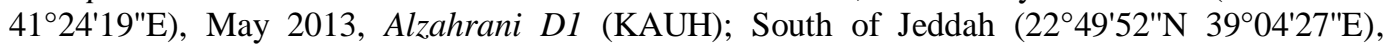
February 2013, Alzahrani et Albokhari D\&E103 (KAUH); $12 \mathrm{~km}$ south of Alleith (19 $50^{\prime} 10^{\prime \prime} \mathrm{N}$ 40³0'07"E), February 2013, Alzahrani D121 (KAUH); Bishah, March 2013, Al Dahhan 2

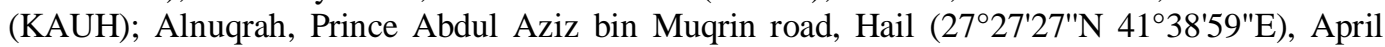


2013, Asiri 2 (KAUH); Wady Fatmah, January 1945, Khattab K 48 (CAIM) Mahazat Al-Said reserve area, July 2002, Al-Abbasi et Shalhoub SAU-13 (K); Musaymir Wadi Tuban, March 1967, Smih et Lawranos 25 (K); Wadi Gdeidat $130 \mathrm{~km}$ northwest of Mecca near Rabeh Saabar et Johfar, May 2004, Al-Abbasi 0220802 (K); Jizan, $10 \mathrm{~km}$ south of Baysh from Sabya just before Wadi Guman, March 1996, Van Slageren et Al-sa'doon 261 (K); Eastern provinces Al-Hasa lower south slope of Jabal Shaban, February 1965, Mandaville 375 (K); Madina road $30 \mathrm{~km}$ north-east from Yanbu junction, March 1977, Collenette 16 (K). QATAR: Qatar Road to Doha, January 1971, Wilcox 57 (K); Wadi Al Galaiel toward the southern end of the Qatar peninsula, April 1977, Boulos H11126 (K); Dukhan road, April 1979, Batanouny 2462 (K). BAHRAIN: Southern Jebeh, May 1979, Virgo 81 (K); Ras Noma, February 1970, Gauaeher 50 (K); western plains of Bahrain main Island, April 1984, Rezk 119 (K). OMAN: Matrah in Wadi behind town, March 1969, Dickson 1095 (K); Sultan Qaboos University campus Seeb, April 1987, Cope 172 (K); Al Hallaniyah, Kuria Muria Islands, November 1993, McLeish 3028 (E). UNITED ARAB EMIRATES: Abu Dhabi in the vicinity of Umm am Nar near to the old Abu Dhabi airport, May 1982, Western 292 (CAI); Persian Gulf, March 1937, Holmes 346 (K); Abu Dhabi, March 1972, Wilcox 207 (K). YEMEN: Aden, Jabal Shamsan tower of Silence and vicinity, June 1987, Boulos et al. 16531 (K); Socotra, 1898, Grant et Expedition 73 (E); Socotra, February 1989, Miller et al. M. 8498 (E). EGYPT: Ismailia-Cairo road, November 1979, Costantin et al. 499 (CAIM). PAKISTAN: Baluchistan, Bela to Uthal, April 1965, Lamond 223 (E); Baluchistan, Makrani; Pasni to Kappan road to Gwadar, April 1965, Lamond 445 (E); Karachi, October 1949, Jafri s.n. (E). INDIA: Punjab, January 1886, Drummond 21622 (E). NAMIBIA: $5.3 \mathrm{~km}$ east of Goageb along road to Keetmanshoop, May 1993, Strohbach 2339 (E); Omaruru District, Uis-Barandberg west road 50 km, April 1987, Long et Rae 757 (E). KENYA: August 1938, Pole Evans et Erens 1608 (E).

Zygophyllum fabago L., Sp. Pl. 1: 385 (1753).

(Fig. 15).

Diagnosis: Z. fabago can be distinguished by its compound leaves, with two flat, obovateelliptical leaflets, glabrous ovary and oblong loculicidal capsule.
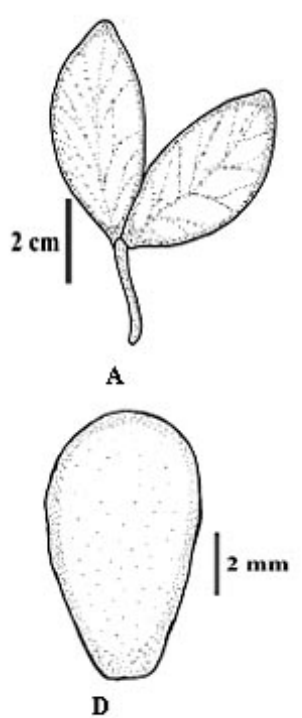

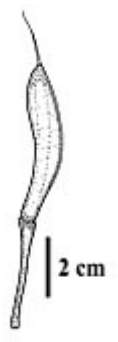

B

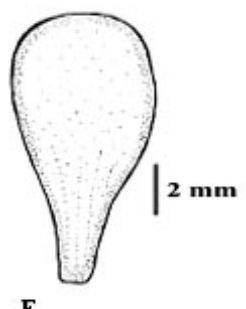

$\mathbf{E}$

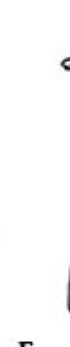

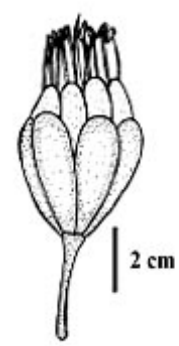

c

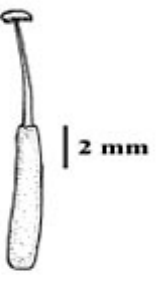

G

Fig. 15. Zygophyllum fabago: A. Leaf; B. Fruit; C. Flower; D. Sepal; E. Petal; F. Stamen; G. Ovary. 
Small shrub, perennial, green, up to $75 \mathrm{~cm}$ tall. Leaves 2-foliolate, leaflets flat, obovateelliptic, $40 \times 25 \mathrm{~mm}$, obtuse, petiole $20 \mathrm{~mm}$ long. Flowers bisexual, white-creamy, $12 \times 15 \mathrm{~mm}$, pedice $16 \mathrm{~mm}$ long. Sepals 5 , yellowish green, obovate-elliptic, $10 \times 5 \mathrm{~mm}$, aestivation imbricate. Petals 5, white, $12 \times 5 \mathrm{~mm}$, aestivation valvate. Stamens 10 , c. $13 \mathrm{~mm}$ long, staminal appendages undivided, $6 \times 1 \mathrm{~mm}$; anthers 2-lobed, yellow, dorsifixed, dehiscent longitudinally. Ovary glabrous; style single, $6.5 \mathrm{~mm}$ long. Fruit a loculicidal, oblong-cylindrical capsule, c. $30 \times(5) 10 \mathrm{~mm}$, glabrous, peduncle up to $10 \mathrm{~mm}$ long.

Vernacular names: Rotreyt, Qyllab.

Distribution: Saudi Arabia: Northern region (Tabarjal) (Fig. 16). Worldwide: Egypt, Palestine, Syria, Jordan, Iraq, Iran, Pakistan, Turkey, Spain, Georgia, Armenia, Russia, Afghanistan, Azerbaijan, France and Armenia.

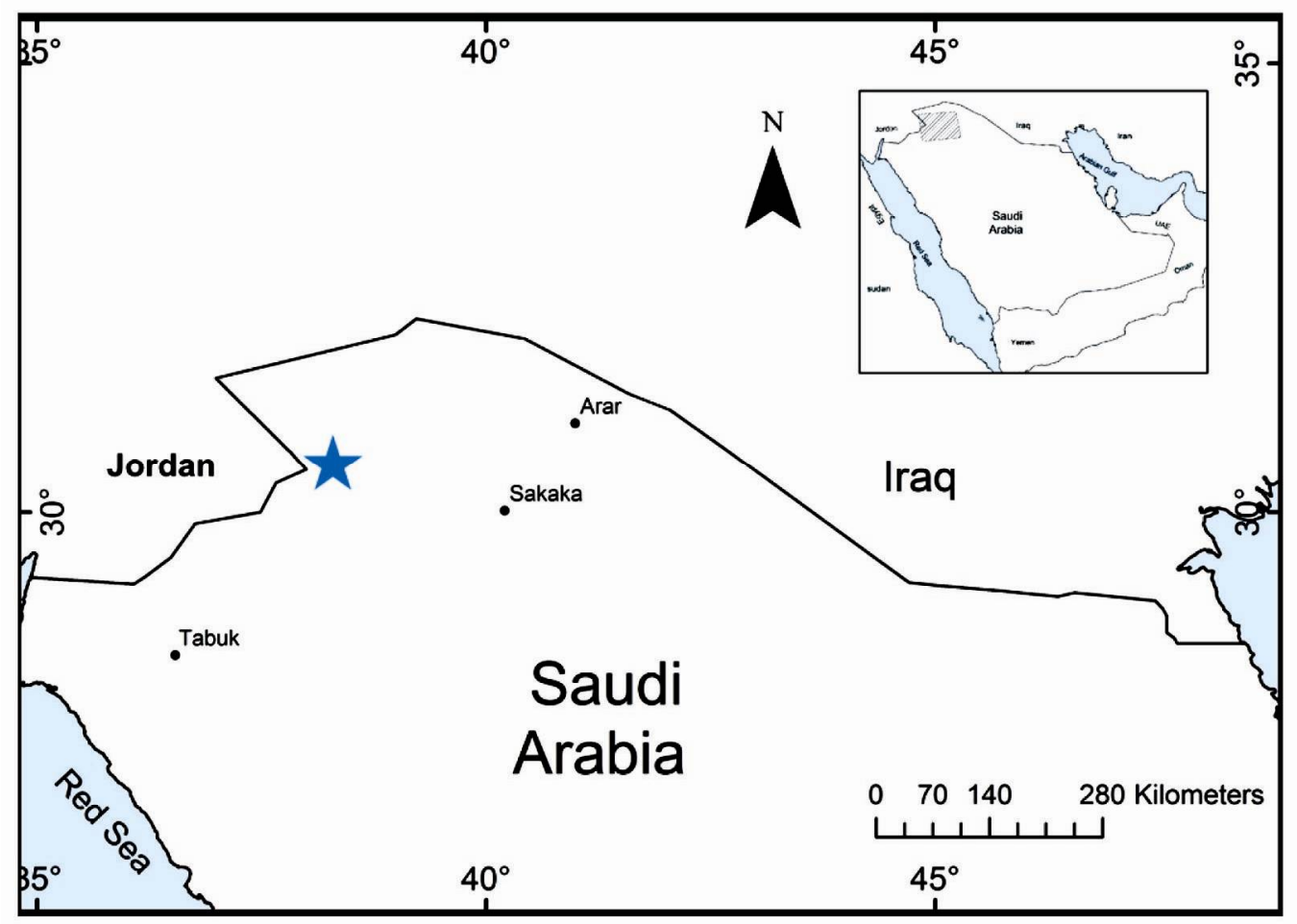

Fig. 16. Distribution map of Zygophyllum fabago in Saudi Arabia.

Habitat: Sandy soils.

Specimens examined: SAUDI ARABIA: Al Asawia $10 \mathrm{~km}$ before Tabarjal, April 1988, Alaadin, Al Yahya et Al Said H19038 (RIY). EGYPT: Wadi Qoseib, December, 1944, Alhabetai Z6477 (CAIM). IRAQ: Abu Ghraib, June 1959, Rawi 26935 (CAIM); Baghdad, near Saadun State, October 1954, Haines 72 (E); Baghdad Liwa, May 1956, Polunin et Duri 68 (E). IRAN: Kazvin in Ditione Oppindikeredj, September 1948, Rechinger 6841 (E); Azerbaijan, Moghan, bank of Aras River, $40 \mathrm{~km}$ from Parsabad on road to Aslanduz, May 1971, Lamond 3182 (E); Azerbaijan south of Khoi, July 1960, Furse et Synge 799/2 (E). JORDAN: Azrag, April 1936, Dinsmore 11805 (E). SYRIA: July 1890, Post s.n. (E); June 1910, Haradjian 3406 (E); Palmyra small dunes at north end of seat lake, April 1943, Davis 5900 (E). TURKEY: Kars, Iğdir, state 
Breeding Farm, around Boralar tepesi (hill), July 1956, Demiris 3301 (E); Vily er Agri, October 1910, Post 2053 (E); Prov. Sivas, east of Susehri, August 1957, Davis et Hedge 32706 (E). GEORGIA: Davit Gorgeji, at caves, August 2009, Mitchell et al. 52 (E); Tbilisi, hill sides near Dababane gorge, opposite the Tbilisi Botanical Institute, June 1959, Davis 33708 (E); URSSGéorgie-Tbilisi, July 1978, Leonard 7155 (E). SPAIN: Coastal steppe in the hills between La Unión and Cartagena, April 1957, Stud. biol. Rheno-Trai 57-535 (CAI); Almeria, Velez Blanco, roadside $10 \mathrm{~km}$ west of the village $\left(37^{\circ} 38^{\prime} \mathrm{N} 2^{\circ} 6^{\prime} \mathrm{E}\right)$, July 1981, Gardner et Gardner 1501 (E); Champs incultes prés de Cartagena, July 1852, Bourgeau 2050 (E). RUSSIA: 1900, Kulikowski E (E); July 1896, Callier 51 (E). ARMENIA: Circa ruinas sanctuarii Zvartnoc ad occidentem urbis Ervan, July 1975, Gabrielian 12853 (E). AFGHANISTAN: Tashkurghan, Septiembre 1937, Koelz 13182 (E); Samangan Tangi Taschkurgan Streamside, June 1969, Ekberg 9072 (E); Maymana, halfway between Maymana and Andkhui, June 1962, Hedge et Wendelbo W3834 (E). AZERBAIJAN: Elisabethpol distr. Araeseh Geok-tapa, in ruderatis, May 1908, Schelkownikow et Woronow 312 (E). FRANCE: Héraull, Port de Cette, July 1891, Bernard s.n. (E).

\section{Acknowledgements}

We thank the authority of the herbaria BM, CAI, CAIM, E, K, KAUH, KSU and RIY for giving us access to their collections. We are thankful to Dr. Shahina Ghazanfar of the Royal Botanic Gardens, Kew, London for her assistance during our visit to this herbarium. We are grateful to Mrs. Mona S. Al Harbi for her help in preparing distribution maps, and to Mr. Mohammed Alnaggar for his assistance during field trips.

\section{References}

Al-Arjany, K.M. 2011. Molecular taxonomic perspective and eco-physiological variations of some species of Tribulus, Zygophyllum and Fagonia genera of family Zygophyllaceae in Saudi Arabia. Master Dissertation, King Saud University, Saudi Arabia.

Alzahrani, D.A. 2017. Systematic studies on the Zygophyllaceae of Saudi Arabia: Two new subspecies combination in TetraenaMaxim. Saudi J. Biol. Sci. DOI: 10.1016/j.sjbs.2016.12.022.

Alzahrani, D.A. and Albokhari, E.J. 2017a. Molecular phylogeny of Saudi Arabian Tetraena Maxim. and Zygophyllum L. (Zygophyllaceae) based on plastid DNA sequences. Bangladesh J. Plant Taxon. 24(2): 155-164.

Alzahrani, D.A.and Albokhari, E.J. 2017b. Systematic studies on the Zygophyllaceae of Saudi Arabia: a new variety and new variety combination in Tetraena. Saudi J. Biol. Sci. 24: 1574-1579.

Alzahrani, D.A. and Albokhari, E.J. 2017c. Systematic studies on the Zygophyllaceae of Saudi Arabia: new combinations in Tetraena Maxim. Türk. J. Bot. 41: 96-106.

APG (Angiosperm Phylogeny Group). 2009. An update of the Angiosperm Phylogeny Group classification for the orders and families of flowering plants. Bot. J. Linn. Soc. 161: 105-121.

Azevedo, L.B. 2014. Development and application of stressor-response relationships of nutrients. Ph.D. Thesis, Radboud University Nijmegen, the Netherlands.

Beier, B.A., Chase, M.W. and Thulin, M. 2003. Phylogenetic relationships and taxonomy of subfamily Zygophylloideae (Zygophyllaceae) based on molecular and morphological data. Plant Syst. Evol. 240: 11-39.

Chaudhary, S.A. 2001. Flora of the Kingdom of Saudi Arabia, Vol. 3. Ministry of Agriculture and Water, Riyadh, Saudi Arabia.

Collenette, S. 1985. An Illustrated Guide to the Flowers of Saudi Arabia. Scorpion Publishing, London, pp. 506-507.

Collenette, S. 1998. A Checklist of Botanical Species in Saudi Arabia. International Asclepiad Society, Ashford: Headley Brothers Ltd. 
Collenette, S. 1999. Wild Flowers of Saudi Arabia. NCWCD, Kingdom of Saudi Arabia, pp. 764-766.

Cronquist, A. 1968. The Evolution and Classification of Flowering Plants. Boston: Houghton Mifflin, 396 pp.

Dahlgren, R. 1980. A revised system of classification of the angiosperms. Bot. J. Linn. Soc. 80: 91-124.

Endlicher, S.L. 1841. Genera Plantarum Secundum Ordines Naturales Disposita. Part 18: 1161. Vienna: Fr. Beck.

Engler, A. 1931. Zygophyllaceae. In: Engler, A. and Prantl, K. (2 ${ }^{\text {nd }}$ ed.), Die Naturlichen Pflanzenfamilien 19: 144-184. Leipzig: Engelmann.

Engler, A. 1964. Syllabus der Pflanzenfamilien. Gebrüder Borntraeger, Berlin Nikolassee, 203 pp.

Ghazanfar, S.A. 2007. Flora of the Sultanate of Oman 2, Crassulaceae - Apiaceae. Scripta Bot. Belg. 36: 1220.

Ghazanfar, S.A. and Osborn, J. 2015. Typification of Zygophyllum propinquum Decne. andZ. coccineum L. (Zygophyllaceae) and a key to Tetraena in SW Asia. Kew Bull. 70: 1-9.

Hadidi, M.N. 1977. Two new Zygophyllum species from Arabia. Publications from Cairo University Herbarium 7 \& 8: 327-329.

Hadidi, M.N. 1978. Zygophyllaceae. In: Boulos, L. (Ed.), Materials for a flora of Qatar. Webbia 32: 369-396.

Hosny, A.I. 1988. Genus Zygophyllum L. in Arabia. Taeckholmia 11: 19-32.

Hutchinson, J. 1969. Evolution and Phylogeny of Flowering Plants. Dicotyledons: Facts and theory with over 550 illustrations and maps by the author. London, New York: Academic Press.

IUCN 2014. The IUCN Red List of Threatened Species, version 2014.1 Cambridge UK: IUCN Red List Unit, Available from: http:// www.iucnredlist.org (accessed on 20.12.2014).

Linnaeus, C. 1753. Species Plantarum. Holmiae: Impensis Laurentii Salvii, pp. 385-386.

Louhaichi, M., Salkini, A.K., Estita, H.E. and Belkhir, S. 2011. Initial assessment of medicinal plants across the Libyan Mediterranean coast. Adv. Environ. Biol. 5: 359-370.

Mandaville, J.P. 1990. Flora of Eastern Saudi Arabia. London and New York: Kegoin Pul International Ltd.

Migahid, A.M. 1978. Flora of Saudi Arabia, Vol. 1, Seconded. Riyadh University Publications, Riyadh, Saudi Arabia.

Migahid, A.M. 1996. Flora of Saudi Arabia, Vol. 1, Fourthed. Riyadh University Publications, Riyadh, Saudi Arabia.

Mosti, S., Raffaelli, M. and Tardelli, M. 2012. Contribution to the flora of Central-Southern Dhofar (Sultanate of Oman). Webbia 67: 65-91.

Norton, J., Abdul Majid, S., Allan, D., AlSafran, M., Böer, B. and Richer, R. 2009. An Illustrated Checklist of the Flora of Qatar. Browndown Publications, Gosport, UK, 67 pp.

Oltmann, O. 1971. Pollenmorphologisch-systematische untersuchungen innerhalb der Geraniales. Dissertation, Berlin.

Sakkir, S., Kabshawi, M. and Mehairbi, M. 2012. Medicinal plants diversity and their conservation status in the United Arab Emirates (UAE). J. Med. Plant Res. 6: 1304-1322.

Sheahan, M.C. and Chase, M.W. 1996. A phylogenetic analysis of Zygophyllaceae based on morphological, anatomical and $r b c$ L DNA sequence data. Bot. J. Linn. Soc. 122: 279-300.

Sheahan, M.C. and Chase, M.W. 2000. Phylogenetic relationships within Zygophyllaceae based on DNA sequences of three plastid regions, with special emphasis on Zygophylloideae. Syst. Bot. 25: 371-384.

Soliman, M.S., El-Tarras, A.S. and El-Awady, M.A. 2010. Seed exomorphic characters of some taxa from Saudi Arabia. J. Amer. Sci. 6: 906-910.

Symanczik, S., Blanzkowski, J., Koegel, S., Boller, T., Wiemken, A. and Al-Yahya'ei, M. 2014. Isolation and identification of desert habituated arbuscular mycorrhizal fungi newly reported from the Arabian Peninsula. J. Arid Land 6(4): 488-497.

Takhtajan, A.L. 1980. Outline of the classification of flowering plants (Magnoliophyta). Bot Rev. 46: 225-359.

Takhtajan, A.L. 1987. Systema Magnoliophytorum. Leningrad, Nauka. 
Van Huyssteen, D.C. 1937. Morphologisch-systematische Studien über die Gattung Zygophyllum. Dissertation. Berlin.

Van Zyl, L. 2000. A systematic revision of Zygophyllum in the southern African region. Ph.D. thesis, University of Stellenbosch, Stellenbosch.

Waly, N.M., Al-Ghamdi, F.A. and Al-Shamrani, R.I. 2011. Developing methods for anatomical identification of the genus Zygophyllum L. (Zygophyllaceae) in Saudi Arabia. Life Sci. 8: 451-459.

Zumbruch, H.J. 1931. Über die Bedeutung des Saponins für die systematische Gliederung der Zygophyllaceen-Gattungen. Dissertation, Berlin.

(Manuscript received on 13 January 2018; revised on 10 March 2018) 